\title{
Ist die zunehmende Biogasproduktion die alleinige Ursache für den Grünlandschwund in Deutschland? - eine Analyse von georeferenzierten Daten zur Landnutzung
}

\author{
Birgit Laggner • Natascha Orthen • \\ Bernhard Osterburg • Norbert Röder
}

Eingegangen: 31. Juli 2013 / Angenommen: 6. Februar 2014 / Online publiziert: 25. März 2014

(C) Springer-Verlag Berlin Heidelberg 2014

\begin{abstract}
Zusammenfassung In den letzten Jahren ist die Grünlandfläche in Deutschland stark zurückgegangen. Dieser Rückgang wird von vielen Seiten kritisch gesehen, da Grünland im Vergleich zum Ackerland in Bezug auf Kriterien des Umwelt- und Naturschutzes besser bewertet wird. In der öffentlichen Diskussion wird der Rückgang des Grünlandes unter anderem mit der Zunahme des Maisanbaus aufgrund der Förderung der Biogasproduktion im Rahmen des Erneuerbaren-Energien-Gesetzes (EEG) in Verbindung gebracht. In diesem Beitrag wird ein Geoinformationssystem vorgestellt, das verschiedene hoch aufgelöste Daten zur Landnutzung, zu Landnutzungsänderungen und Standortbedingungen verarbeitet. Mithilfe dieses Systems wird für den Zeitraum von 1999 bis 2007 untersucht, ob die Biogaserzeugung die Hauptursache für den beobachteten Landnutzungswandel ist. Die Analyse erfolgt für Deutschland als Ganzes sowie mit einem höher aufgelösten Datenbestand für Niedersachsen (2005-2007), das ein Zentrum der Biogaserzeugung ist. Die Ergebnisse zeigen, dass der Anbau von Gärsubstraten vor allem auf schon bestehenden Ackerflächen erfolgt. In Betrieben mit Anbau
\end{abstract}

\footnotetext{
N. Röder $(\varangle) \cdot$ B. Laggner $\cdot$ N. Orthen $\cdot$ B. Osterburg

Institut für Ländliche Räume, Johann Heinrich von Thünen-Institut, Bundesforschungsinstitut für Ländliche Räume, Wald und Fischerei, Bundesallee 50, 38116 Braunschweig, Deutschland

E-Mail: norbert.roeder@ti.bund.de

B. Laggner

E-Mail: birgit.laggner@ti.bund.de

N. Orthen

E-Mail: natascha.orthen@ti.bund.de

B. Osterburg

E-Mail: bernhard.osterburg@ti.bund.de
}

von Gärsubstraten wird Grünland eher umgebrochen, allerdings bewirtschaften diese Betriebe nur sehr wenige ehemalige Grünlandflächen. Mit abnehmender Entfernung zur nächsten Biogasanlage steigt die Wahrscheinlichkeit, dass Grünland umgewandelt wird. Der Großteil der Umwandlung von Grünland in Ackerflächen erfolgt allerdings durch Betriebe, die keine Gärsubstrate anbauen. Hier sind insbesondere intensiv wirtschaftende Milchviehbetriebe zu nennen. Grünland wird vor allem dann umgewandelt, wenn der Bewirtschafter der Fläche wechselt. Auf der Basis der Ergebnisse werden verschiedene Ansätze zum Schutz des Dauergrünlandes im Speziellen und zur Begrenzung von direktem und indirektem Landnutzungswandel im Allgemeinen diskutiert.

Schlüsselwörter Landnutzungswandel · Bioenergie · Landwirtschaft · Hoch aufgelöste Daten

\section{Is the Increasing Bio-methane Production the Sole Reason for the Declining Grassland Area in Germany? An Analysis of Geo-referenced Land Use Data}

\begin{abstract}
In the last years the area used as permanent grassland declined considerably in Germany. This decline raised public concerns as grassland produces more environmental benefits compared to arable land. In the public debate the loss of grassland is frequently linked to the promotion of silage maize as feedstock for bio-methane production via the renewable energy law. We present a geo information system, which is capable to process various high resolution data on land use, land use change and site conditions. With the help of the system we analyse whether the expansion of bio-methane production is the primary reason for the observed land use change in the period 1999-2007.
\end{abstract}


We provide results for Germany as a whole. These general results are supplemented by an analysis conducted for the federal state of Lower Saxony (2005-2007). This state is a stronghold of the bio-methane production and more detailed data are available. Our results show, that feedstock for the production of bio-methane is primarily cultivated on existing arable land. Farms growing feedstock for biomethane production have a higher probability to convert grassland to arable land though these farms cultivate only very few areas of former grassland. The probability of grassland conversion increases with the vicinity of biogas plants. However, most of the conversion can be attributed to dairy farms without the cultivation of feedstock for bio-methane production. Generally, the likelihood that grassland is converted increases markedly if the managing farmer changes. Based on our findings we discuss different options to protect permanent grassland. We argue that our conclusions do also apply to the general context of limiting undesired direct and indirect land use change.

Keywords Land use change $\cdot$ Bioenergy $\cdot$ Agriculture $\cdot$ High resolution data

\section{Einleitung}

In den letzten 15 Jahren werden zunehmend Informationen zu Landnutzung, Umweltparametern und Schutzgebietskulissen auch für größere Räume georeferenziert bereitgestellt. Gerade Landnutzungsänderungen können anhand publizierter statistischer Daten zur landwirtschaftlichen Flächennutzung nur begrenzt nachvollzogen werden, da in der Regel Flächensalden ausgewiesen werden. Rückschlüsse auf die Wanderungen zwischen den einzelnen Nutzungsklassen und die Brutto-Flächenveränderungen sind auf der Grundlage solcher aggregierter Daten nicht möglich. Der vorliegende Beitrag zeigt am Beispiel der Entwicklung der Grünlandfläche auf, welche Daten für eine detaillierte Analyse der landwirtschaftlichen Flächennutzung zur Verfügung stehen und wie diese genutzt werden können.

Sowohl für die Erklärung und Prognose von Änderungen in der landwirtschaftlichen Landnutzung als auch für die Bewertung dieser Änderungen ist eine Vielzahl von räumlich hoch aufgelösten Daten notwendig. Die absolute und relative Wirtschaftlichkeit der verschiedenen landwirtschaftlichen Produktionsverfahren hängt stark von den natürlichen Standortbedingungen (Klima, Boden, Relief) ab. Insbesondere Boden und Relief können kleinräumig sehr stark variieren. Ferner spielen Transportentfernungen, selbst wenn es sich um Distanzen von wenigen Kilometern handelt, bei der Festlegung der Flächennutzung innerhalb des Betriebes eine wichtige Rolle. Dies gilt insbesondere, wenn im landwirtschaftlichen Betrieb wasserreiche Subst- rate wie Gülle (Wassergehalt $>90 \%$ ) oder Silagen (Wassergehalt $>65 \%$ ) transportiert werden. Außerdem beschränken Schutzgebiete mit ihren Auflagen den Entscheidungsspielraum der Landwirte. Die Bewertung einer Landnutzung bzw. einer Landnutzungsänderung kann nicht ohne zusätzliche Informationen zum jeweiligen Standort vorgenommen werden. So hängt die Wirkung einer Grünlandumwandlung in Ackerland auf die Freisetzung von Treibhausgasen von den jeweiligen Bodenparametern ab (Poeplau/Don/Vesterdal et al. 2011).

Dem Erhalt von Dauergrünland wird aus einer Vielzahl von Gründen eine hohe Priorität in der Diskussion der ökologischen Auswirkungen der Landwirtschaft eingeräumt. So gehören extensiv bewirtschaftete Grünländer zu den artenreichsten Kulturlandschaften in Europa (Dierschke/ Briemle 2002: 10, 196; Stoate/Baldi/Beja et al. 2009: 31). Die Wiederherstellung artenreicher Bestände ausgehend von Ackerland oder intensiven Grünlandbeständen dauert oft sehr lange (vgl. Bakker/Berendse 1999). Im Vergleich zu Ackerland ist Grünland meist durch höhere Kohlenstoffvorräte im Boden gekennzeichnet (IPCC 2000: 13). Insbesondere organische Böden wie Moore speichern sehr große Kohlenstoffmengen (Freibauer/Drösler/Gensior et al. 2009: 20; Lal 2008: 817). Die Umwandlung von Grünland zu Ackerland führt zu einer verstärkten Mineralisation der organischen Bodensubstanz. Für mineralische Standorte wird von einem Rückgang der organischen Bodensubstanz um ein Drittel im Pflughorizont (oberste $30 \mathrm{~cm}$ ) ausgegangen (Johnston/Poulton/Coleman 2009: 26; Poeplau/Don/Vesterdal et al. 2011: 2421). Auf organischen Böden ist der Verlust des Bodenkohlenstoffs eine Funktion des mittleren Grundwasserflurabstandes, der auf Ackerflächen meist deutlich unter dem Niveau von Grünlandflächen liegt (Drösler/Adelmann/Augustin et al. 2013: 97 f.). Des Weiteren haben Äcker eine geringe Fähigkeit zur Wasserspeicherung und Nährstoffrückhaltung, sodass es zu einer stärkeren Auswaschung von Nährstoffen in Oberflächenund Grundwasser kommen kann (Nitsch/Osterburg/von Buttlar et al. 2008: 3; Schramek/Osterburg/Kasperczyk et al. 2012: 3) und die Erosionsgefährdung ist höher (EEA 2007: 46; Nitsch/Osterburg/von Buttlar et al. 2008: 3, 25 f.). Die Umwandlung von Grünland zu Ackerland führt damit zu einer Einschränkung verschiedener Ökosystemleistungen. Das Ausmaß der jeweiligen Einschränkung ist aber stark standortabhängig.

Auch von Seiten der Agrarpolitik wird der Bedeutung des Grünlandschutzes Rechnung getragen. So bestehen sowohl für die abgelaufene als auch die künftige Förderperiode der EU sogenannte Cross Compliance-Auflagen, die den Umfang der zulässigen Grünlandumwandlung begrenzen (Europäische Kommission 2009). Zusätzlich erfolgt eine Förderung der Grünlandnutzung über Agrarumweltmaßnahmen im Rahmen der ländlichen Entwicklungsprogramme. 
Der Umfang der Grünlandfläche geht in Deutschland seit mehreren Jahrzehnten zurück. Zwischen 1991 und 2012 nahm die Dauergrünlandfläche um 0,7 Mio. ha auf 4,6 Mio. ha ab, während sich im gleichen Zeitraum der Umfang der Ackerfläche um 0,3 Mio. ha auf 11,8 Mio. ha vergrößerte. ${ }^{1}$ Gleichzeitig nahm die Siedlungs- und Verkehrsfläche stark zu (Statistisches Bundesamt 2013). Aus dieser aggregierten Betrachtung kann nicht gefolgert werden, dass primär Grünland in Siedlungs- und Verkehrsfläche umgewandelt wird. Vielmehr überlagern sich zwei Phänomene: die Umwandlung von Grün- in Ackerland und Die Überführung von Landwirtschaftsflächen in andere Nutzungen, vor allem in Siedlungs- und Verkehrsfläche. In der Hälfte der Jahre seit 1991 ging die Grünlandfläche um weniger als $0,3 \%$ pro Jahr zurück. Deutlich höhere Raten von über 1\% pro Jahr waren nur zwischen 1998 und 2001 sowie zwischen 2007 und 2010 zu beobachten. Da in den Jahren 1999 und 2010 die Mindestgröße für die statistisch erfassten landwirtschaftlichen Betriebe erhöht wurde, sind kleinere Betriebe aus der Erfassung gefallen. Die in diesen Zeiträumen ausgewiesenen, erhöhten Grünlandrückgänge sind daher auch auf diese methodischen Änderungen der statistischen Erhebung zurückzuführen.

In den letzten Jahren wird der Grünlandrückgang zunehmend mit einem erhöhten Umwandlungsdruck aufgrund steigender Agrarpreise und der Förderung der nachwachsenden Rohstoffe zur Energieerzeugung in Verbindung gebracht (DVL/NABU 2007; Roskam 2007; Greiff/WeberBlaschke/Faulstich et al. 2010). Die Förderung des Anbaus nachwachsender Rohstoffe ist hierbei einer der Gründe für die in den letzten Jahren steigenden Preise für landwirtschaftliche Produkte. Einen Überblick hierzu geben Kretschmer/Bowyer/Buckwell (2012). Trotz einer Vielzahl an negativen Auswirkungen auf Natur und Landschaft wird die Nutzung erneuerbarer Energien vor allem in strukturschwachen ländlichen Regionen als ökonomische Chance betrachtet (Kosfeld/Gückelhorn 2012: 445).

Eine Diskussion über direkte und indirekte Landnutzungsänderungen aufgrund geänderter Politiken erfolgt seit Searchinger/Heimlich/Houghton et al. (2008) vor allem mit dem Fokus auf die Effekte von Politiken zur Förderung des Einsatzes von Bioenergie und die Klimaeffizienz solcher Maßnahmen. Hierbei ist anzumerken, dass nahezu jede Maßnahme, die zu einer höheren Nachfrage nach landwirtschaftlichen Produkten oder zu einer Verringerung der produzierten Mengen (z. B. Ausweitung des Öko-Landbaus) führt, neben direkten auch indirekte Landnutzungsänderungen impliziert. Führt eine Politik unmittelbar zu einer Umwidmung der Nutzung einer bestimmten Fläche,

\footnotetext{
${ }^{1}$ Daten des Statistischen Bundesamtes, verschiedene Jahrgänge der Fachserie 3, Reihe 3 ,Landwirtschaftliche Bodennutzung und pflanzliche Erzeugung. Land- und Forstwirtschaft, Fischerei“".
}

so bezeichnet man dies als direkte Landnutzungsänderung. $\mathrm{Zu}$ indirekten Landnutzungsänderungen kommt es dann, wenn sich aufgrund einer Politik die relative Wettbewerbsfähigkeit (sprich die Preisrelationen) auch für nicht unmittelbar von der Politik betroffene Betriebe und ihre Produkte verändert.

In Deutschland wird im Zusammenhang mit dem Grünlandumbruch oft der Anbau von Gärsubstraten (Mais) für Biogasanlagen kritisch diskutiert (Pölking/Stiepel/PremkeKraus 2006; WBGU 2009). Diese Diskussion basiert neben regionalen Fallstudien (z. B. Hübner 2010) und Einzelfallbeobachtungen auf mehreren allgemeinen Befunden. Erstens wurde insbesondere der Anbau von Mais zur Gewinnung von Gärsubstraten in den von 2004 bis 2011 gültigen Versionen des Erneuerbaren-Energien-Gesetzes (EEG) stark gefördert, da Mais im Vergleich zu anderen Kulturen komparative Kostenvorteile für die Biogaserzeugung auf der Basis nachwachsender Rohstoffe hat (vgl. de Witte 2012). In diesem Zeitraum kam es zu einer sehr starken Ausweitung des Silomaisanbaus in Deutschland (+0,78 Mio. ha), obwohl der Rinderbestand weiter rückläufig war $(-633.000$ Stück). ${ }^{2}$ Zweitens war in dieser Zeit ein besonders starker Rückgang des Grünlandes zu beobachten, auch in Regionen mit hoher und stabiler Rinderbesatzdichte (Schramek/ Osterburg/Kasperczyk et al. 2012: 7 ff.). Drittens sind die Ansprüche an den Standort von Silomais und intensivem Grünland ähnlich. Beispielsweise finden sich beide Nutzungen auf Standorten mit vergleichsweise hohem Grundwasserstand (Nitsch/Osterburg/von Buttlar et al. 2008: 95). Es stellt sich allerdings die Frage, in wie weit diese Koinzidenz der Ereignisse auf eine Kausalität zurückzuführen ist.

Eine unmittelbare Kausalität besteht im Fall direkter Landnutzungsänderungen, also wenn umgebrochenes Grünland für den Anbau von Gärsubstraten genutzt wird. Allerdings sind auch mittelbare Effekte vorstellbar. So verringert der Anbau von nachwachsenden Rohstoffen die für die Nahrungs- und Futtermittelproduktion zur Verfügung stehende Ackerfläche und führt zu höheren Pachtpreisen (Habermann/Breustedt 2011; Emmann/Theuvsen 2012). Gleichzeitig werden Betriebe mit Gärsubstratanbau aufgrund der Förderung durch das EEG gegenüber anderen Betrieben wettbewerbsfähiger. Die steigenden Preise können dazu führen, dass Betriebe, die keine Gärsubstrate anbauen, Ackerflächen abgeben müssen, da sie auf dem Pachtmarkt nicht mehr konkurrenzfähig sind. Diese Betriebe können nun ihrerseits Grünland umwandeln, um anschließend Ackerfrüchte anzubauen (indirekte Landnutzungsänderung). Daher fordern unter anderen Gawel/ Ludwig (2011: 237) die Berücksichtigung indirekter Landnutzungsänderungen bei der Steuerung von Bioenergie.

\footnotetext{
${ }^{2}$ Silomais ist insbesondere bei der Rinderfütterung ein bedeutendes Futtermittel
} 
Um mehr Klarheit in die Diskussion zwischen der Expansion des Maisanbaus und dem Grünlandrückgang zu bringen, wurde von den Autoren dieses Beitrags ein Geoinformationssystem aufgebaut, das alle verfügbaren, georeferenzierbaren und für die Analyse und Bewertung der landwirtschaftlichen Flächennutzung relevanten Datengrundlagen vereinigt. Auf dessen Basis wird analysiert, inwieweit die räumlichen Zentren des Grünlandrückgangs und der Ausdehnung des Maisanbaus korrelieren und inwieweit ein räumlicher Zusammenhang zwischen dem Betrieb von Biogasanlagen und der Wahrscheinlichkeit der Grünlandumwandlung gegeben ist. Ferner wird untersucht, welche Betriebe Grünland umwandeln und auf welchen Standorten dies erfolgt.

Im Kap. 2 wird das Geoinformationssystem kurz beschrieben. Am Beispiel der Analyse der Wechselwirkung von Biogasförderung und Grünlandumwandlung werden die verwendeten Datenquellen dargestellt und die Auswertungsmethoden skizziert. Die Darstellung der Ergebnisse erfolgt in Kap. 3. Abschließend werden die Ergebnisse diskutiert (Kap. 4).

\section{Material und Methoden}

\subsection{Datengrundlagen}

Mit einem Geoinformationssystem ist es möglich, Daten aus mehreren Themenbereichen zur Analyse der Landnutzung und deren Veränderungen zusammenzuführen. Die Daten beschreiben Landnutzung, natürliche Standortfaktoren, Gebiete mit rechtlichen Nutzungseinschränkungen und administrative Grenzen (vgl. Tab. 1). Die Datensätze unterscheiden sich in ihren Inhalten, ihrer Ausdehnung (Deutschland, einzelne Bundesländer), ihrer räumlichen und thematischen Auflösung, dem Datenformat sowie ihrer Aktualität bzw. Aktualisierungsfrequenz (Zeitreihenfähigkeit).

Für die Beschreibung der Landnutzung werden drei verschiedene Datensätze genutzt. Der aus der Agrarstatistik abgeleitete Datensatz basiert auf der Agrarstrukturerhebung, in der die landwirtschaftliche Flächennutzung und Tierhaltung erfasst werden. Im Gegensatz zu den Veröffentlichungen der Statistischen Ämter enthält dieser Datensatz eine vollständige und konsistente Schätzung der Umfänge der wichtigsten landwirtschaftlichen Produktionsverfahren auf Gemeindeebene. Da die Ausweisung von Tabellenwerten mit wenigen Beobachtungen strengen Datenschutzbestimmungen unterliegt, sind agrarstatistische Daten auf Gemeindeebene wenn überhaupt, dann nur sehr lückenhaft verfügbar. Deshalb wird ein Schätzverfahren eingesetzt, das unter Wahrung des Datenschutzes eine sehr gute Näherung an die erhobene Statistik ermöglicht (Röder/Gocht 2013).
Die Aktualisierung folgt dem Zyklus der Vollerhebungen der Agrarstruktur, die alle drei bis vier Jahre durchgeführt werden. Daten liegen für die Jahre 1999, 2003 und 2007 vor. Die Daten der Agrarstatistik werden an den Forschungsdatenzentren des Bundes und der Länder ausgewertet. Dieser Zugang erlaubt die Analyse von Landnutzungsänderung auf der Basis von einzelbetrieblichen Daten. Die Zuordnung der Flächeninformationen zu einer Gemeinde basiert auf dem Betriebssitzprinzip, das heißt, eine Fläche wird der Gemeinde zugewiesen, in der der jeweilige Landwirtschaftsbetrieb gemeldet ist. Eine Verschneidung mit räumlich wesentlich höher aufgelösten Geofachdaten und deren Analyse ist aufgrund der vergleichsweise ungenauen Abbildung auf Gemeindeebene nur begrenzt sinnvoll.

Den beiden anderen Datensätzen liegen eigene GISGeometrien zugrunde, die eine flächengenaue Abbildung der Landnutzung erlauben. Die Daten des Integrierten Verwaltungs- und Kontrollsystems (InVeKoS) sind aufgrund ihrer hohen räumlichen und inhaltlichen Auflösung die wichtigste Datengrundlage für die landwirtschaftliche Flächennutzung. Sie enthalten zum einen Geometrien der landwirtschaftlichen Flächen in hoher Genauigkeit aus dem „System zur Identifizierung landwirtschaftlicher Parzellen“ ( $< \pm 1$ m; Europäischer Rat 2009; JRC 2012) und zum anderen Informationen zur Flächennutzung, Tierhaltung und zu flächenbezogenen Förderprogrammen. Diese Daten stammen aus den Förderanträgen der Landwirtschaftsbetriebe, die jährlich im Rahmen der EU-Agrarpolitik gestellt werden. Der Flächenidentifikator (FLIK) ${ }^{3}$ beschreibt georeferenziert die zugrundeliegende Geometrie. Die Informationen zur landwirtschaftlichen Nutzung werden seit 2005 jährlich aktualisiert. Die InVeKoS-Daten ermöglichen eine Analyse der landwirtschaftlichen Nutzung nach dem Belegenheitsprinzip, das heißt, es liegen flächenscharfe Informationen zur tatsächlichen Lage der Fläche vor. Die Nutzung dieser Daten ist durch Datenschutzauflagen und den prioritären Zweck, die Verwaltung der Agrarförderung, stark beschränkt.

Das Digitale Basis-Landschaftsmodell (Basis-DLM) ergänzt diese Daten durch flächendeckende Informationen. Ins Geographische Informationssystem (GIS) wurden die Jahre 2000, 2005 und 2010 aufgenommen. Die inhaltliche Auflösung ist allerdings deutlich geringer als bei den vorangestellten Datenquellen. So werden nur Acker- und Grünland sowie bestimmte Sonderkulturen unterschieden, nicht aber einzelne Ackerkulturen (z. B. Weizen, Raps). Angaben zur Tierhaltung fehlen systembedingt vollständig. Auf der anderen Seite werden auch Flächen abgebildet, die von Betrieben bewirtschaftet werden, die unterhalb der statis-

\footnotetext{
${ }^{3}$ Die Abkürzung FLIK wird im Folgenden auch als Bezeichnung der im System zur Identifizierung landwirtschaftlicher Parzellen dargestellten Flächeneinheiten verwendet.
} 
Tab. 1 Übersicht über die verwendeten Datenquellen

\begin{tabular}{|c|c|c|c|c|c|c|}
\hline Datensatz & Autor/Quelle & Inhalt & Format & Maßstab & Aktualisierung & Datenschutz \\
\hline \multicolumn{7}{|l|}{ Landnutzung } \\
\hline $\begin{array}{l}\text { Agrarstrukturerhe- } \\
\text { bung }\end{array}$ & $\begin{array}{l}\text { Gocht/Röder } \\
(2011)\end{array}$ & $\begin{array}{l}\text { Landwirtschaft } \\
\text { (Kulturen und Tier- } \\
\text { haltung), bezogen auf } \\
\text { Gemeinden }\end{array}$ & $\begin{array}{l}\text { Attribut- } \\
\text { Tabelle }\end{array}$ & & $\begin{array}{l}\text { Vorliegende Jahre: } \\
\text { 1999, 2003, } 2007\end{array}$ & $\begin{array}{l}\text { Freigabe der Daten durch } \\
\text { Forschungsdatenzentren der } \\
\text { Länder }\end{array}$ \\
\hline $\begin{array}{l}\text { Integriertes Verwal- } \\
\text { tungs- und Kontroll- } \\
\text { system (InVeKoS) }\end{array}$ & Länder ${ }^{\mathrm{d}, \mathrm{fh}, \mathrm{k}, \mathrm{n}, \mathrm{p}, \mathrm{r}}$ & $\begin{array}{l}\text { Landwirtschaft- } \\
\text { liche Kulturen } \\
\text { (eingeschränkte } \\
\text { Tierhaltung) }\end{array}$ & Vektor & $>1: 10.000$ & $\begin{array}{l}\text { Einzeljahre aus } \\
\text { dem Zeitraum } \\
\text { 2003-2010 (bun- } \\
\text { deslandspezifisch) }\end{array}$ & $\begin{array}{l}\text { Projektgebundene } \\
\text { Nutzungsverträge }\end{array}$ \\
\hline $\begin{array}{l}\text { Digitales Basis- } \\
\text { Landschaftsmodell } \\
\text { (Basis-DLM) }\end{array}$ & $\mathrm{BKG}$ & $\begin{array}{l}\text { Allgemeine Land- } \\
\text { nutzung (Siedlung, } \\
\text { Vegetation, Verkehr } \\
\text { etc.) }\end{array}$ & Vektor & $1: 250.000$ & $\begin{array}{l}\text { Verwendete Jahre: } \\
\text { 2000, 2005, } 2010\end{array}$ & $\begin{array}{l}\text { Ohne } \\
\text { Nutzungseinschränkungen }\end{array}$ \\
\hline \multicolumn{7}{|c|}{ Naturräumliche Standortfaktoren } \\
\hline $\begin{array}{l}\text { Digitales } \\
\text { Höhenmodell }\end{array}$ & BKG & $\begin{array}{l}\text { Höhe, Neigung, } \\
\text { Exposition }\end{array}$ & Raster & $25 \times 25 \mathrm{~m}$ & Stand: 03/2009 & $\begin{array}{l}\text { Ohne } \\
\text { Nutzungseinschränkungen }\end{array}$ \\
\hline Klimadaten & DWD & $\begin{array}{l}\text { Langjährige Monats- } \\
\text { mittel (Tempera- } \\
\text { tur, Niederschlag, } \\
\text { Sonnenscheindauer) }\end{array}$ & Raster & $1 \times 1 \mathrm{~km}$ & $\begin{array}{l}\text { Periode } \\
1978-2009\end{array}$ & $\begin{array}{l}\text { Projektgebundener } \\
\text { Nutzungsvertrag }\end{array}$ \\
\hline $\begin{array}{l}\text { Bodenübersichtskar- } \\
\text { te (BÜK 1.000) }\end{array}$ & BGR & Boden & Vektor & $1: 1.000 .000$ & Erstellung 1995 & $\begin{array}{l}\text { Ohne } \\
\text { Nutzungseinschränkungen }\end{array}$ \\
\hline $\begin{array}{l}\text { Bodenübersichtskar- } \\
\text { te (BÜK 200) }\end{array}$ & BGR & Boden & Vektor & $1: 200.000$ & Stand: $05 / 2010$ & Nutzungsvertrag \\
\hline $\begin{array}{l}\text { Mittelmaßstäbige } \\
\text { Landwirtschaftliche } \\
\text { Standortkartierung } \\
\text { (MMK 100) }\end{array}$ & Länder ${ }^{\mathrm{a}, \mathrm{g}}$ & Boden & Vektor & $\begin{array}{l}1: 25.000- \\
1: 100.000\end{array}$ & $\begin{array}{l}\text { Zum Teil alte } \\
\text { Daten (18./19. } \\
\text { Jahrhundert) }\end{array}$ & $\begin{array}{l}\text { Projektgebundene } \\
\text { Nutzungsverträge }\end{array}$ \\
\hline $\begin{array}{l}\text { Bodenübersichtskar- } \\
\text { te bzw. Bodenkarte } \\
\text { (BÜK 50, BK 50) }\end{array}$ & Länder ${ }^{\mathrm{i}, \mathrm{l}}$ & Boden & Vektor & $1: 50.000$ & $\begin{array}{l}\text { Stand der Daten } \\
\text { variiert zwischen } \\
1996 \text { und } 2001\end{array}$ & $\begin{array}{l}\text { Projektgebundene } \\
\text { Nutzungsverträge }\end{array}$ \\
\hline $\begin{array}{l}\text { Geologische Über- } \\
\text { sichtskarte (GÜK } \\
\text { 200) }\end{array}$ & BGR & $\begin{array}{l}\text { Oberflächengeologie } \\
\text { ( } 2 \mathrm{~m} \text { Tiefe) } \\
\text { (inkl. Torfe) }\end{array}$ & Vektor & $1: 200.000$ & Stand: 2003 & Nutzungsvertrag \\
\hline Moorkarten & Länder ${ }^{\mathrm{c}, \mathrm{g}}$ & $\begin{array}{l}\text { Moorkataster, sensib- } \\
\text { le Moore etc. }\end{array}$ & Vektor & $\begin{array}{l}1: 5.000- \\
1: 50.000\end{array}$ & $\begin{array}{l}\text { Stand der Daten } \\
\text { variiert zwischen } \\
1998 \text { und } 2008\end{array}$ & $\begin{array}{l}\text { Projektgebundene Nut- } \\
\text { zungsverträge/ohne } \\
\text { Nutzungseinschränkungen } \\
\text { (bundeslandspezifisch) }\end{array}$ \\
\hline Wertvolle Biotope & $\begin{array}{l}\text { Länder }{ }^{\mathrm{c}, \mathrm{e}, \mathrm{g}, \mathrm{j}, \mathrm{m}, \mathrm{o}, \mathrm{q}} \\
\text { BfN }\end{array}$ & $\begin{array}{l}\text { Biotoptypen, FFH- } \\
\text { Lebensraumtypen }\end{array}$ & Vektor & $\begin{array}{l}1: 5.000 \\
1: 10.000\end{array}$ & $\begin{array}{l}\text { Stand der Daten } \\
\text { variiert zwischen } \\
2004 \text { und } 2011\end{array}$ & $\begin{array}{l}\text { Projektgebundene Nut- } \\
\text { zungsverträge/ohne } \\
\text { Nutzungseinschränkungen } \\
\text { (bundeslandspezifisch) }\end{array}$ \\
\hline \multicolumn{7}{|c|}{ Rechtliche Nutzungseinschränkungen } \\
\hline Schutzgebiete & $\mathrm{BfN}$ & $\begin{array}{l}\text { Schutzgebiete nach } \\
\text { Bundesnaturschutz- } \\
\text { gesetz }\end{array}$ & Vektor & $1: 25.000$ & Stand: $01 / 2008$ & Nutzungsvertrag \\
\hline Wasserschutzgebiete & Länder ${ }^{c, e, g, j, m, o, q}$ & $\begin{array}{l}\text { Trinkwasser, Heil- } \\
\text { quellen etc. }\end{array}$ & Vektor & $\begin{array}{l}1: 25.000 \\
1: 75.000\end{array}$ & $\begin{array}{l}\text { Stand der Daten } \\
\text { variiert zwischen } \\
2007 \text { und } 2011\end{array}$ & $\begin{array}{l}\text { Projektgebundene Nut- } \\
\text { zungsverträge/ohne } \\
\text { Nutzungseinschränkungen } \\
\text { (bundeslandspezifisch) }\end{array}$ \\
\hline $\begin{array}{l}\text { Überschwemmungs- } \\
\text { gebiete }\end{array}$ & Länder ${ }^{\mathrm{b}, \mathrm{e}, \mathrm{g}, \mathrm{j}, \mathrm{m}, \mathrm{o}, \mathrm{r}}$ & $\begin{array}{l}\text { 100-jährige } \\
\text { Überflutungsbereiche }\end{array}$ & Vektor & $<1: 5.000$ & $\begin{array}{l}\text { Stand der Daten } \\
\text { variiert zwischen } \\
2004 \text { und } 2012\end{array}$ & $\begin{array}{l}\text { Projektgebundene Nut- } \\
\text { zungsverträge/ohne } \\
\text { Nutzungseinschränkungen } \\
\text { (bundeslandspezifisch) }\end{array}$ \\
\hline $\begin{array}{l}\text { Wasserrahmenricht- } \\
\text { linie }\end{array}$ & Länder ${ }^{\mathrm{c}, \mathrm{j}, \mathrm{r}}$ & Kulissen & Vektor & $\begin{array}{l}1: 10.000 \\
1: 25.000\end{array}$ & $\begin{array}{l}\text { Stand der Daten } \\
\text { variiert zwischen } \\
2009 \text { und } 2011\end{array}$ & $\begin{array}{l}\text { Projektgebundene Nut- } \\
\text { zungsverträge/ohne } \\
\text { Nutzungseinschränkungen } \\
\text { (bundeslandspezifisch) }\end{array}$ \\
\hline
\end{tabular}


Tab. 1 (Fortsetzung)

\begin{tabular}{|c|c|c|c|c|c|c|}
\hline Datensatz & Autor/Quelle & Inhalt & Format & Maßstab & Aktualisierung & Datenschutz \\
\hline \multicolumn{7}{|l|}{$\overline{\text { Sonstiges }}$} \\
\hline $\begin{array}{l}\text { Verwaltungsgebiete } \\
\text { (VG 250) }\end{array}$ & $\mathrm{BKG}$ & $\begin{array}{l}\text { Verwaltungseinheiten } \\
\text { auf Gemeindeebene }\end{array}$ & Vektor & $1: 250.000$ & Stand: 2008 & $\begin{array}{l}\text { Ohne } \\
\text { Nutzungseinschränkungen }\end{array}$ \\
\hline Biogasanlagen & $\begin{array}{l}\text { Bundesnetz- } \\
\text { agentur }\end{array}$ & $\begin{array}{l}\text { Strommengen, Anla- } \\
\text { gengröße, Substrate }\end{array}$ & Punkt & $\begin{array}{l}\text { Adress- } \\
\text { daten (ohne } \\
\text { Maßstab) }\end{array}$ & $\begin{array}{l}\text { Verwendete Jahre: } \\
\text { 2009, } 2010\end{array}$ & $\begin{array}{l}\text { Ohne } \\
\text { Nutzungseinschränkungen }\end{array}$ \\
\hline
\end{tabular}

$B K G$ Bundesamt für Kartographie und Geodäsie, $D W D$ Deutscher Wetterdienst, $B G R$ Bundesanstalt für Geowissenschaften und Rohstoffe, BfN Bundesamt für Naturschutz, FFH-Lebensraumtypen Lebensraumtypen nach Anhang I der Fauna-Flora-Habitatrichtlinie (FFH-Richtlinie vom 21. Mai 1992, 92/43/EWG)

${ }^{a}$ Landesamt für Bergbau, Geologie und Rohstoffe Brandenburg

${ }^{\mathrm{b}}$ Landesumweltamt Brandenburg

'Ministerium für Ländliche Entwicklung, Umwelt und Verbraucherschutz Brandenburg

'Ministerium für Infrastruktur und Landwirtschaft des Landes Brandenburg

'Bayerisches Landesamt für Umwelt

${ }^{\mathrm{f} B a y e r i s c h e s ~ S t a a t s m i n i s t e r i u m ~ f u ̈ r ~ E r n a ̈ h r u n g, ~ L a n d w i r t s c h a f t ~ u n d ~ F o r s t e n ~}$

${ }^{g}$ Landesamt für Umwelt, Naturschutz und Geologie Mecklenburg-Vorpommern

${ }^{\text {h} M i n i s t e r i u m ~ f u ̈ r ~ L a n d w i r t s c h a f t, ~ U m w e l t ~ u n d ~ V e r b r a u c h e r s c h u t z ~ M e c k l e n b u r g-V o r p o m m e r n ~}$

${ }^{i}$ Landesamt für Bergbau, Energie und Geologie Niedersachsen

${ }^{j}$ Niedersächsischer Landesbetrieb für Wasserwirtschaft, Küsten- und Naturschutz

${ }^{\mathrm{k} S e r v i c e z e n t r u m ~ L a n d e n t w i c k l u n g ~ u n d ~ A g r a r f o ̈ r d e r u n g ~ b e i ~ d e r ~ B e h o ̈ r d e ~ f u ̈ r ~ G e o i n f o r m a t i o n, ~ L a n d e n t w i c k l u n g ~ u n d ~ L i e g e n s c h a f t e n ~(G L L) ~}$ Hannover

'Geologischer Dienst Nordrhein-Westfalen

${ }^{m}$ Landesamt für Natur, Umwelt und Verbraucherschutz Nordrhein-Westfalen

${ }^{n}$ Ministerium für Klimaschutz, Umwelt, Landwirtschaft, Natur- und Verbraucherschutz Nordrhein-Westfalen

${ }^{\circ}$ Landesamt für Umwelt, Wasserwirtschaft und Gewerbeaufsicht Rheinland-Pfalz

${ }^{\mathrm{p} M i n i s t e r i u m}$ für Wirtschaft, Verkehr, Landwirtschaft und Weinbau des Landes Rheinland-Pfalz

${ }^{q}$ Landesamt für Landwirtschaft, Umwelt und ländliche Räume des Landes Schleswig-Holstein

${ }^{r}$ Ministerium für Landwirtschaft, Umwelt und ländliche Räume des Landes Schleswig-Holstein

tischen Erfassungsgrenze liegen (Agrarstatistik) bzw. von Betrieben, die keine Agrarförderung erhalten (InVeKoSV 2004; Osterburg/Nitsch/Laggner et al. 2009). Daneben ermöglicht das Digitale Basis-Landschaftsmodell die Ableitung von Aussagen zur Landnutzung und Landnutzungsänderungen auf nicht-landwirtschaftlich genutzten Flächen (Siedlungs- und Verkehrsflächen, Wald etc.).

Herausforderungen bei der Auswahl und Beschaffung weiterer, georeferenzierter Fachdaten betreffen sowohl den Zugang zu diesen Geofachdaten als auch deren Vergleichbarkeit bezüglich Auflösung und Informationsinhalt. In Deutschland existieren bedingt durch die föderale Verwaltungsstruktur keine länderübergreifenden Standards zur Erhebung, Aufbereitung und Weitergabe von Geofachdaten. Aus diesem Grund müssen Informationen für einen so entscheidenden Faktor wie den Boden aus den unterschiedlichsten Quellen abgeleitet werden. Die Spanne reicht von der deutschlandweiten Bodenübersichtskarte im Maßstab 1:1.000.000 (BÜK 1.000) bis zu Bodenkarten im Maßstab 1:50.000. Daneben spielt die geologische Übersichtskarte (GÜK 200) insbesondere bei der Abgrenzung von organischen Böden eine wichtige Rolle. Geofachdaten zur
Lage von Schutz- und Überschwemmungsgebieten liegen dagegen in stärker vereinheitlichter Form vor.

Weiterhin sind in das System deutschlandweite Daten für Biogasanlagen zur räumlichen Lage (Punktgeometrien, georeferenziert auf der Grundlage der gelieferten Adressdaten), Anlagenleistung, erzeugten Energiemenge und zu eingesetzten Substraten (laut Vergütungsschlüssel) eingepflegt. Diese privatwirtschaftlichen Daten werden jährlich von der Bundesnetzagentur im Rahmen der Berichterstattung zur Umsetzung des EEG bereitgestellt. Die Auswahl der Biogasanlagen aus den veröffentlichten Daten erfolgt auf der Basis des Vergütungsschlüssels (Substratkategorien, Anlagenleistung).

\subsection{Verwendete Software}

Die Verwaltung der Geodaten erfolgt in einer PostgreSQLDatenbank (PostgreSQL 8.4.5). Bei den InVeKoS-Daten ist aus Gründen des Datenschutzes eine Trennung zwischen Geodaten und Attributdaten (Antragsdaten, Betriebsbezug) erforderlich. Die aus Datenschutzsicht sensiblen Attributdaten werden auf einem gesicherten, stark zugangs- 
beschränkten Server vorgehalten und sind in den Prozess der Geodatenverarbeitung nicht involviert.

Für die vektorbezogene Geodatenverarbeitung werden sowohl die PostgreSQL-Erweiterung PostGIS (Version 1.5.2) als auch ArcGIS 10 verwendet. Python-Skripte für ArcGIS werden mit Python 2.6 bzw. 2.7 unter der Oberfläche von Pythonwin programmiert. Die Rasterdaten werden mit GRASS 6.4 bearbeitet.

Die statistische Auswertung der Daten erfolgt mit SAS 9.3.

\subsection{Verarbeitung der Geodaten}

Ziel der Geodaten-Verarbeitung ist es, die für die Betrachtung von Landnutzung bzw. Landnutzungsänderungen relevanten Informationen aus den gelieferten Geodaten miteinander zu verknüpfen und für die statistische Analyse zur Verfügung zu stellen. Die Verknüpfung erfolgt auf der Basis des räumlichen Bezugs, dessen Herstellung je nach Datentyp des Inputdatensatzes variiert:

a. Vektorflächen (Polygone): Überlagerung (Verschneidung) und Berechnung der Teilfächen mit Zuordnung zu Attributen der Inputdatensätze

b. Rasterdaten, innerhalb von Flächenidentifikatoren: statistische Beschreibung der Verteilung aller Rasterwerte innerhalb eines Polygons

c. Rasterdaten, außerhalb von Flächenidentifikatoren: Ziehung einer gemeinsamen Stichprobe aus verschnittenen Vektorpolygonen (a.) und Rasterkarten in Form eines regelmäßigen Punktrasters

d. Vektorpunkte (Biogasanlagen): Entfernungsberechnung zu Mittelpunkten der Flächenidentifikatoren

Da die meisten Daten in Form von Vektorpolygonen vorliegen, nimmt Variante a. eine zentrale Stellung bei der Geodatenverarbeitung ein. Die Verknüpfung mit anderen Datenformaten (Rasterdaten (b.), Vektorpunkte (d.)) orientiert sich zumeist an den Flächenidentifikatoren, da auf deren Auswertung ein besonderer inhaltlicher Fokus liegt (vgl. Kap. 2.4). Variante c. liefert Informationen über die Gebiete außerhalb der Flächenidentifikatoren. Aufgrund der $\mathrm{zu}$ analysierenden Datenmenge wird nur eine Stichprobe auf der Basis eines 100-m-Punktrasters analysiert.

Vor dem eigentlichen Verknüpfungsschritt ist eine Vorprozessierung der gelieferten Inputdaten erforderlich. Alle Inputdatensätze werden in das Deutsche Hauptdreiecksnetz in der Gauß-Krüger-Projektion für den 3. Meridianstreifen (DHDN Zone 3, EPSG-Code: 31467) als einheitlichem Bezugssystem überführt. Ferner wird geprüft, ob die Attributdaten der Inputdatensätze eindeutig und vollständig den vorhandenen Geometrien zugeordnet werden können.

Für Datensätze, die aus Vektorpolygonen bestehen bzw. das Resultat der Verschneidung von Vektorpolygonen sind,

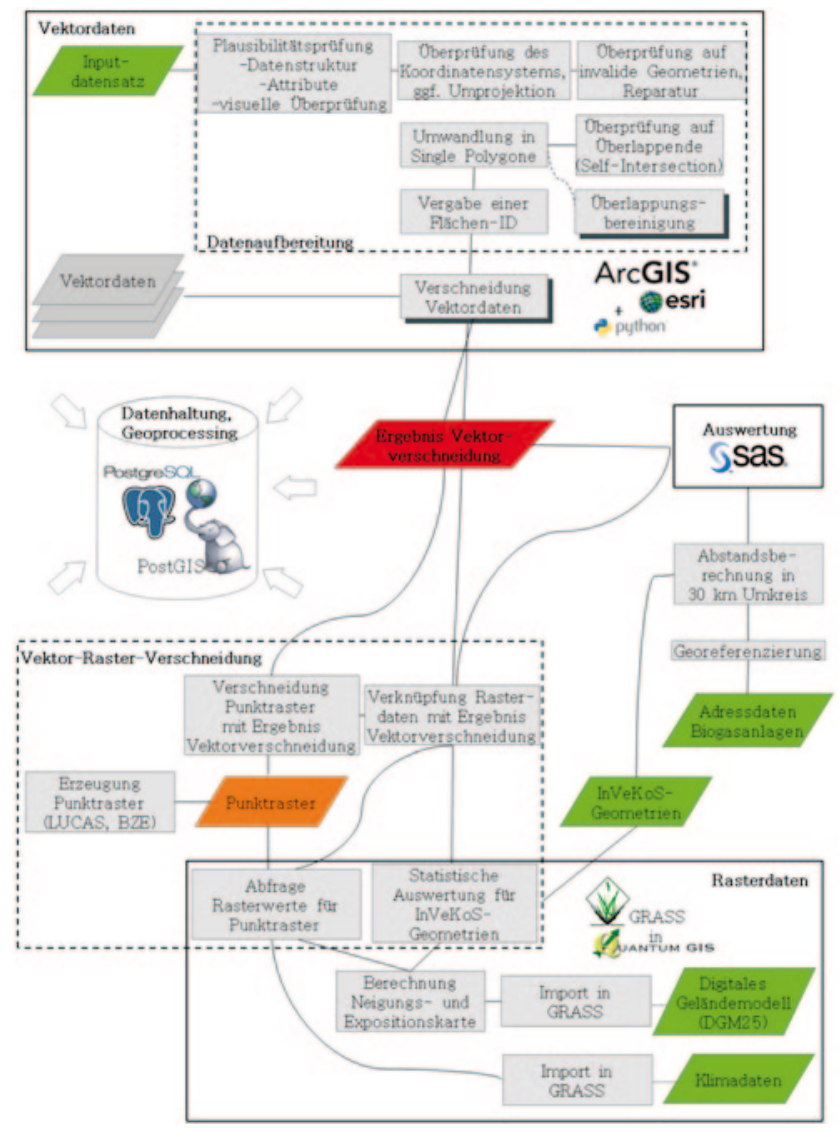

Abb. 1 Ablaufschema der Datenverarbeitung sowie Überblick über die verwendete Software. (Quelle: Verändert nach Laggner/Orthen 2013)

sind weitere Aufarbeitungsschritte nötig. So müssen die Polygone überlappungsfrei sein, da es sonst zu Mehrfachzählungen kommen kann. Ferner müssen alle Inputpolygone bei einer Vektorverschneidung die Validitätsregeln (Prüfen auf invalide Polygone, Umwandlung von Multipolygonen in Singlepolygone) erfüllen, da sie sonst bei der Verschneidung zu Fehlern führen.

Der Ablauf der Geodatenbearbeitung ist in Abb. 1 dargestellt. Eine detaillierte Beschreibung der einzelnen Arbeitsschritte findet sich in Osterburg/Nitsch/Laggner et al. (2009) und Laggner/Orthen (2013).

Eine besondere Herausforderung stellt die Verschneidung der Vektorpolygone dar. Aufgrund der Größe und Anzahl der Inputdatensätze werden die vorhandenen Softund Hardwareressourcen stark beansprucht und die Gefahr von prozessterminierenden Fehlern steigt. Um die Verschneidungsroutine zu entlasten, werden alle Datensätze mit einer Fishnet-Maske in kleinere Pakete zerteilt. Die Verschneidung erfolgt für jedes Paket einzeln und erst nach dem Import in die PostgreSQL-Datenbank werden die Teilergebnisse zu einem Gesamtergebnis zusammengeführt. 


\subsection{Herausforderungen bei der Analyse von} Landnutzungsänderungen

Die drei verwendeten Datenquellen sind unterschiedlich gut als Grundlage für die statistische Analyse von Landnutzungsänderungen in der Landwirtschaft geeignet. Für die Agrarstatistik werden Daten zur Landnutzung schon seit Jahrzehnten erhoben und ausgewertet. Allerdings weisen die offiziellen Statistiken aus Datenschutzgründen oft große Lücken auf, wenn man an Daten auf der Gemeindeebene interessiert ist (vgl. Röder/Gocht 2013). Nur für die Erhebungen von 1999, 2003 und 2007 sind die Daten so aufgearbeitet, dass die Ableitung eines bundesweiten in sich konsistenten Datensatzes möglich ist (Gocht/Röder 2011). Dies ist insofern bedauerlich, als Veränderungen der landwirtschaftlichen Landnutzung nur sehr langsam stattfinden und eine Vollerhebung nur alle drei bis vier Jahre erfolgt. Die räumlich hoch aufgelöste Auswertung von Änderungen der Landnutzung auf Basis der Daten zur Agrarstatistik ist nicht ohne Fallstricke. Die Hauptursache hierfür ist das oben erläuterte Betriebssitzprinzip. Dies hat zur Folge, dass mit Ausnahme des Bundesgebietes keine fixe Flächenkulisse existiert und die Veränderung der landwirtschaftlichen Nutzfläche insgesamt oder einer bestimmten Nutzung in einer Region verschiedene Ursachen haben kann: Erstens können Betriebe (Pacht-)Flächen an Betriebe außerhalb der eigenen administrativen Bezugseinheit (z. B. Gemeinde) abgeben oder sie nehmen Flächen von außerhalb dazu. Zweitens können Betriebe ihren Betriebssitz in eine andere administrative Einheit verlagern. Drittens werden Flächen in eine andere Nutzungsform überführt, z. B. durch Umwandlung in Siedlungsfläche oder Wald. Nur in diesem Fall würde man landläufig von einer Landnutzungsänderung sprechen, in den anderen Fällen bildet die Statistik nach Betriebssitzprinzip keine Nutzungsänderungen ab. Je größer die räumliche Auflösung der Daten ist und je weniger Entscheidungseinheiten betrachtet werden, desto höher ist der Anteil der in der Statistik ausgewiesenen Änderungen der Landnutzung, der durch Verlagerungen von Flächen und Betrieben erklärt werden kann (vgl. das Problem der Varianzinstabilität bei Anselin/Lozano/Koschinsky 2006). Da keine Einzelflächen ausgewertet werden können, liefert die Agrarstatistik nur Netto-Veränderungen, in denen beispielsweise die Grünlandflächengewinne und -verluste in den Landwirtschaftsbetrieben einer Gemeinde saldiert sind. Eine Information über die zugrunde liegenden Brutto-Veränderungen, also getrennt nach Flächengewinnen und -verlusten, fehlt.

Die InVeKoS-Daten werden nach dem Belegenheitsprinzip verwaltet. Somit sind einige der oben genannten Probleme nicht relevant. Allerdings können innerhalb eines Flächenidentifikators verschiedene Kulturen von mehreren unterschiedlichen Betrieben angebaut werden, so dass Flä- chenentwicklungen nur auf der FLIK-Ebene nachvollzogen werden können. Wie bei der Agrarstatistik ist nur eine Nettobetrachtung möglich, allerdings in einer wesentlich genaueren räumlichen Auflösung. Bei der Agrarstatistik werden alle Veränderungen in der Flächennutzung aller Betriebe einer Gemeinde saldiert, während bei einer Analyse der InVeKoS-Daten eine Saldierung je Flächenidentifikator erfolgt. Im Vergleich zur Analyse auf Gemeindeebene (etwa 1.800 ha landwirtschaftliche Nutzfläche je Gemeinde) erhöht sich damit die räumliche Auflösung um mehr als den Faktor 200 (etwa 8 ha landwirtschaftliche Nutzfläche je Flächenidentifikator). Allerdings erfolgt die Verwaltung der Agrarförderdaten im Rahmen eines Geographischen Informationssystems erst seit 2005. Dabei zeigt sich ein Nebeneinander der Neuetablierung von Grünland aus bisherigem Ackerland und der Umwandlung von Grünland in Ackerland. Außerdem spielt die spätere Nichtmeldung von Grünlandflächen, die 2005 noch erfasst waren („Deaktivierung“) sowie die Meldung ,neuer 'Flächen („Aktivierung“) eine Rolle. Die ebenfalls stattfindende Änderung der Flächennutzung zu Bauland kann ohne zusätzliche Daten nicht von einer Nichtmeldung ohne Flächennutzungsänderung unterschieden werden, beides führt zu einem Rückgang der statistisch erfassten Grünlandfläche.

Wie die genannten Veränderungen der Grünlandfläche im InVeKoS-System erfasst werden, ist in Abb. 2 illustriert. Dokumentiert werden nur die im Fördersystem erfassten Flächen. Über die verschiedenen Arten der Flächenveränderung können nur indirekte Schlüsse gezogen werden. Diese beruhen auf einem Vergleich der Meldungen auf FLIKBasis über mehrere Jahre. Eine gleichzeitige Aktivierung und Deaktivierung unterschiedlicher Teilflächen eines Flächenidentifikators kann genauso wenig erfasst werden wie ein Flächentausch zwischen Acker- und Grünland. Berücksichtigt werden muss außerdem, dass auch das Ackerland und andere landwirtschaftliche Nutzflächen einer Aktivierung und Deaktivierung unterliegen können und dass es Umwandlung zwischen Grünland und anderer landwirtschaftlicher Nutzflächen (z. B. Dauerkulturen) geben kann. Aus Gründen der Vereinfachung werden die entsprechenden Flächenveränderungen in Abb. 2 aber nicht dargestellt.

\subsection{Methode}

Zur Abschätzung der Flächenumfänge, auf denen Grünland in Ackerland umgewandelt wird, müssen zunächst die Aktivierungs- und Deaktivierungsprozesse anhand der Flächenveränderungen im Flächenidentifikator identifiziert werden. Eine Zunahme des Grünlands in einem Flächenidentifikator bei gleichzeitiger Zunahme der in den Agraranträgen für diesen Flächenidentifikator insgesamt gemeldeten Fläche wird als Aktivierung eingeordnet, eine Flächenabnahme sowohl von Grünland als auch der 
Abb. 2 Exemplarische Bilanzierung der Grünlandflächen und ,Sichtbarkeit‘ der Flächenveränderungen im InVeKoS-System (mit Brutto-Flächenänderungen in Hektar für Niedersachsen, 2005-2007)

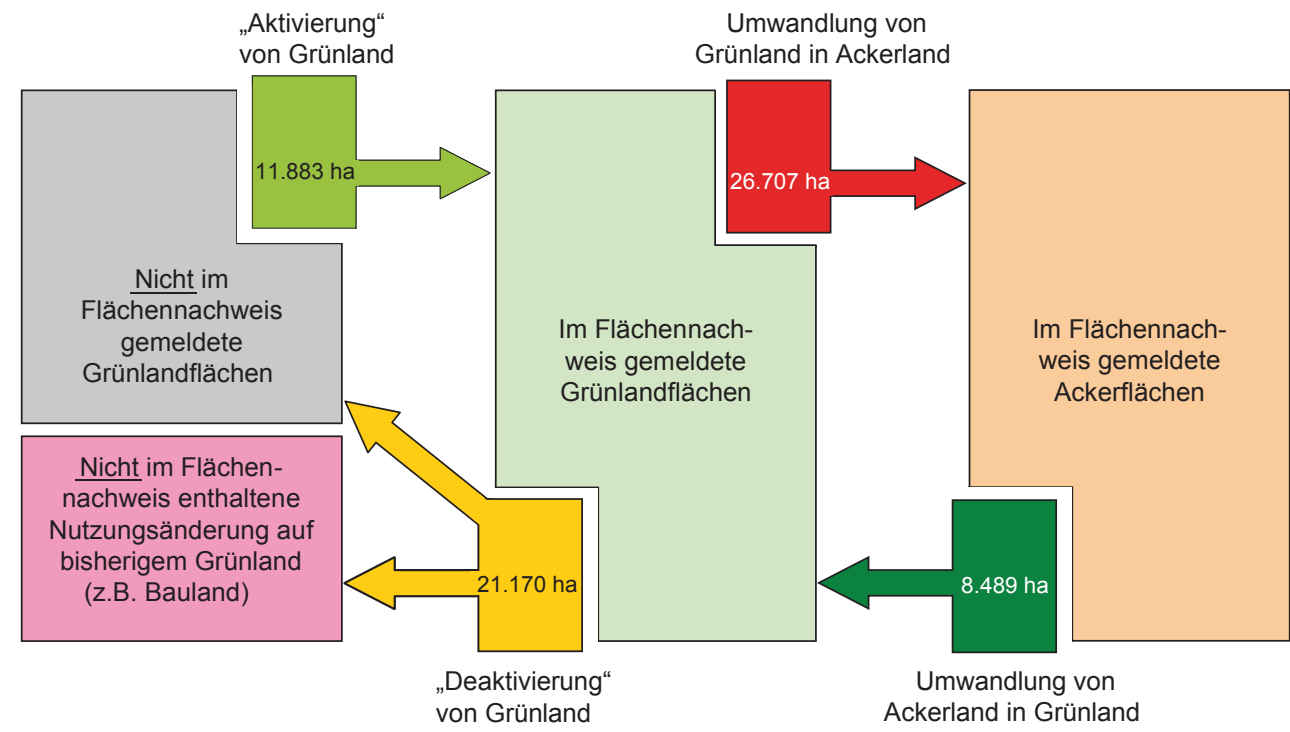

Antragsfläche als Deaktivierung. Entsprechend wird auch im Falle von Zunahmen oder Verlusten von Ackerland bei gleichzeitiger Veränderung der landwirtschaftlichen Nutzfläche vorgegangen. Verbleibende Flächenveränderungen ohne Änderung der landwirtschaftlichen Nutzfläche lassen sich nun der Umwandlung von Grünland in Ackerland (Abnahme von Grünland $=$ Zunahme von Ackerland im Flächenidentifikator) oder der Umwandlung von Ackerland in Grünland (Abnahme von Ackerland = Zunahme von Grünland im Flächenidentifikator) zuordnen.

Am Beispiel von Niedersachsen werden die Möglichkeiten, die sich durch die Analyse von InVeKoS-Daten ergeben, aufgezeigt. Die kleinste räumliche Einheit dieser Daten ist der Feldblock. Ein Feldblock ist durch mehr oder minder fest umgrenzte Flächen (z. B. Wege, Waldränder, Gräben) gekennzeichnet. Er kann jedoch von verschiedenen Betrieben bewirtschaftet werden, die unterschiedliche Kulturen anbauen. Aufgrund der Förderbedingungen lässt sich der Gärsubstratanbau für die Biogasproduktion im InVeKoS nur von 2005 bis 2007 eindeutig identifizieren. Von 2004 bis 2007 verdoppelte sich die Zahl der Anlagen und die installierte elektrische Leistung verdreifachte sich (ML/MU 2012).

Hierzu zählen Maisflächen mit Energiepflanzenprämie oder mit Anbau als nachwachsender Rohstoff auf Stilllegungsfläche. Die auf Grundlage der InVeKoS-Daten gebildeten Betriebsgruppen mit und ohne Gärsubstratanbau werden mithilfe des Wilcoxon-Rangsummentests auf signifikante Unterschiede hinsichtlich der Grünlandumwandlungsrate überprüft. Der Gärsubstratanbau und die Grünlandumwandlung werden anhand eines Logit-Modells auf Zusammenhänge mit der Entfernung (Luftlinie) zur nächsten Biogasanlage untersucht. In dieses Modell gehen ferner der Bodentyp und der Schutzstatus der Flächen ein.
Im Gegensatz zur Agrarstatistik und zu den InVeKoSDaten beschreibt das Digitale Landschaftsmodell seit 2000 die Flächennutzung vollständig. Ein Problem bei der statistischen Analyse von Änderungen ist, dass es keine exakten Stichtage gibt, deren Stand das Digitale Landschaftsmodell widerspiegelt, sondern dass die Daten kontinuierlich ungefähr im Drei- bis Fünf-Jahres-Rhythmus aktualisiert werden. Ferner ist es auf der Basis des Digitalen Basis-Landschaftsmodells nicht möglich, Rückschlüsse zu ziehen, wer Landnutzungsänderungen durchgeführt hat.

\section{Ergebnisse}

In einem ersten Schritt wird das Problem der Umwandlung von Grünland in Ackerland bei gleichzeitiger Ausweitung des Maisanbaus anhand der Daten der Agrarstatistik beleuchtet. Zwischen 1999 und 2007 nahm die Fläche des Dauergrünlandes in Deutschland um über 0,26 Mio. ha ab. Das Zentrum des Rückganges war Nordwestdeutschland (vgl. Abb. 3). Hier ging der Anteil des Grünlandes an der landwirtschaftlichen Nutzfläche flächendeckend um mehr als $2,5 \%$ zurück. Weitere, etwas kleinere Zentren lagen im Schwarzwald und im Alpenraum. Größere Gebiete mit einer nennenswerten Zunahme des Grünlandanteils gab es nur in Hessen. Im Betrachtungszeitraum stieg die mit Mais bestellte Fläche um 0,30 Mio. ha an. In den Gebieten mit einer flächigen Abnahme des Grünlandanteils erfolgte oft eine Ausweitung des Maisanbaus. Eine Ausnahme sind hier nur der Schwarzwald und der Alpenraum. Allerdings nahm der Maisanbau in einem Gebiet vom östlichen Niedersachsen bis zum westlichen Brandenburg und von Mecklenburg bis ins nördliche Sachsen-Anhalt stark zu, ohne dass dort der Anteil des Grünlandes stark zurückging. Zwischen der Entwicklung der Flächenanteile von Grünland und 
Abb. 3 Veränderung des Anteils von Grünland und Mais an der landwirtschaftlichen Nutzfläche zwischen 1999 und 2007. (Quelle: Eigene Auswertung basierend auf dem Datensatz von Röder/ Gocht (2013))
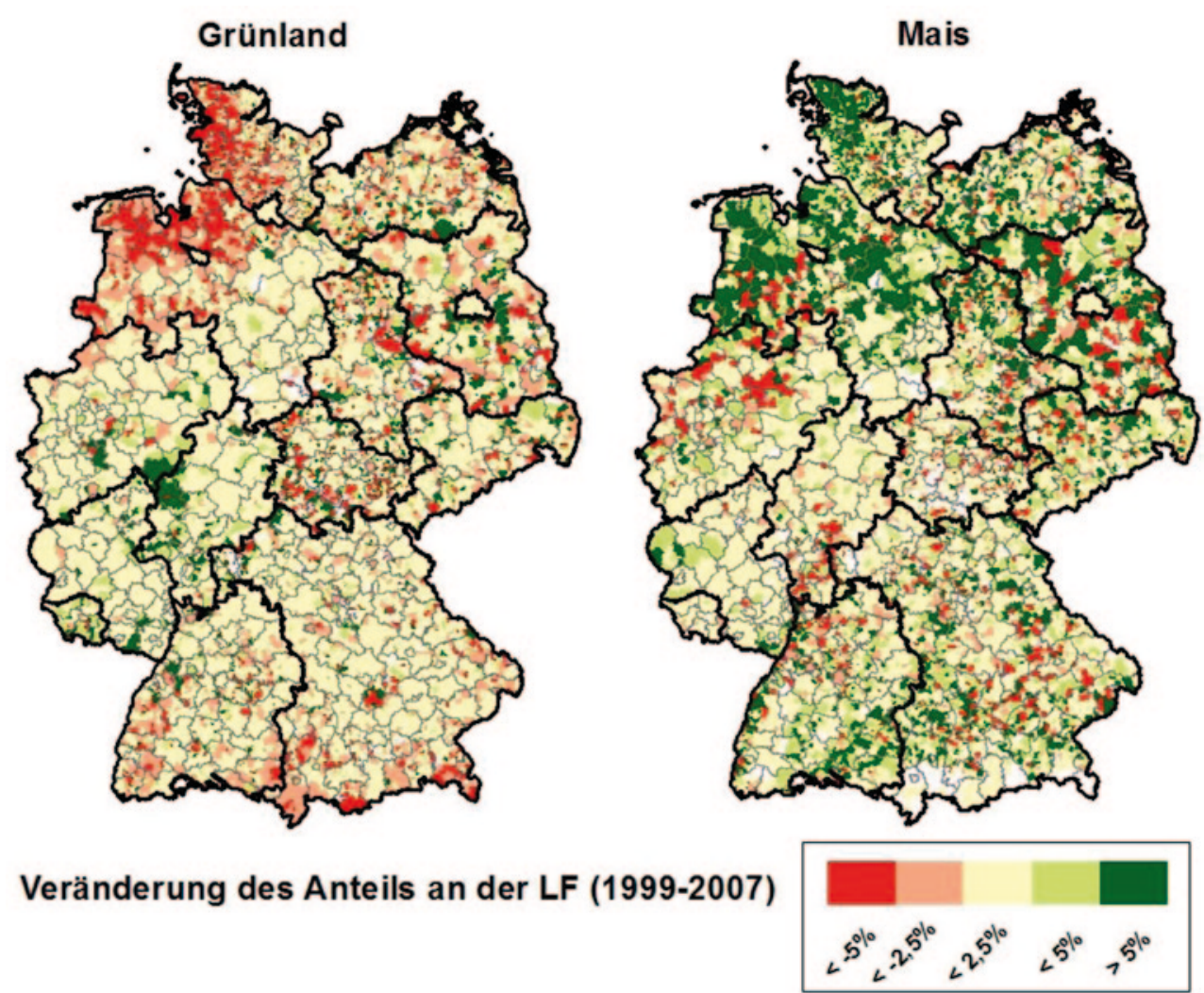

Veränderung des Anteils an der LF (1999-2007)
Mais besteht kein eindeutiger Zusammenhang. In 28\% der Gemeinden nahm sowohl der Anteil des Grünlandes als auch der mit Mais bestellten Fläche ab, während in 19\% der Gemeinden beide Anteile zunahmen. Allerdings gibt es so gut wie keine Zunahme der Grünlandfläche in Gemeinden, wenn der Maisanteil um mehr als 5\% zunahm.

Da im Osten Deutschlands meist sehr wenige Betriebe ihren Sitz innerhalb derselben Gemeinde haben und sich die von ihnen bewirtschafteten Flächen über mehrere Gemeinden erstrecken, ergibt sich hier kein eindeutiges räumliches Muster. Aufgrund der Eigenschaften des Datensatzes sind somit keine Aussagen möglich, welche Nutzungen aufeinander folgen.

Betrachtet man die Entwicklung aller landwirtschaftlichen Betriebe, die über den gesamten Zeitraum von 1999 bis 2007 bestehen, so fällt auf, dass in diesen Betrieben die Grünlandfläche leicht zunimmt, der Grünlandanteil aber nahezu konstant bleibt (vgl. Tab. 2). Der Grünlandanteil in den ausscheidenden Betrieben (34\%) liegt deutlich über dem Ausgangswert der fortgeführten Betriebe. Der Grünlandanteil kann bei den fortgeführten Betrieben nur dann konstant bleiben, wenn die Umwandlung des Grünlands insbesondere bei der Übergabe der Flächen im Rahmen der Betriebsaufgabe erfolgt. In den Betrieben mit Substratanbau wird erst nach 2003 der Maisanbau sehr stark ausgedehnt (im Schnitt um+17\%) und die absolute Grünlandfläche nimmt in dieser Gruppe ab (vgl. DBFZ 2011).
Tab. 2 Agrarstrukturelle Indikatoren landwirtschaftlicher Betriebe differenziert nach ausgeschiedenen Betrieben sowie Betrieben mit Anbau von Gärsubstraten in 2007. (Quelle: Forschungsdatenzentren der Statistischen Ämter des Bundes und der Länder, AFiD-Panel Agrarstruktur 1999-2007, eigene Berechnungen)

\begin{tabular}{lllll}
\hline Jahr & Kenngröße & $\begin{array}{l}\text { Ausge- } \\
\text { schiedene } \\
\text { Betriebe }\end{array}$ & $\begin{array}{l}\text { Ohne Anbau } \\
\text { Gärsubstrat }\end{array}$ & $\begin{array}{l}\text { Mit Anbau } \\
\text { Gärsubstrat }\end{array}$ \\
\hline 1999 & $\begin{array}{l}\text { Flächengröße } \\
\text { (LF) }\end{array}$ & 14 & 42 & 98 \\
& $\begin{array}{l}\text { Grünlandanteil } \\
\text { (DGL/LF) }\end{array}$ & $34 \%$ & $29 \%$ & $17 \%$ \\
& $\begin{array}{l}\text { Maisanteil } \\
\text { (Mais/AF) }\end{array}$ & $11 \%$ & $14 \%$ & $14 \%$ \\
$\begin{array}{l}\text { Viehbesatz } \\
\text { (GV/LF) }\end{array}$ & 0,76 & 0,84 & 0,75 \\
$\begin{array}{l}\text { Flächengröße } \\
\text { (LF) }\end{array}$ & 0 & 45 & 111 \\
$\begin{array}{l}\text { Grünlandanteil } \\
\text { (DGL/LF) }\end{array}$ & - & $29 \%$ & $15 \%$ \\
$\begin{array}{l}\text { Maisanteil } \\
\text { (Mais/AF) }\end{array}$ & - & $14 \%$ & $26 \%$ \\
$\begin{array}{l}\text { Viehbesatz } \\
\text { (GV/LF) }\end{array}$ & - & 0,77 & 0,65 \\
\hline
\end{tabular}

$L F$ Landwirtschaftliche Fläche, $A F$ Ackerfläche, $D G L$ Dauergrünland, $G V$ Großvieheinheiten

Die niedersächsischen InVeKoS-Daten erlauben eine Schichtung aller Landwirtschaftsflächen in Feldblöcke ohne und mit Grünlandumwandlung in Ackerland. In Abb. 4 wird 
Abb. 4 Veränderung der Maisanbaufläche in Feldblöcken ohne und mit Grünlandumwandlung in Ackerland (Niedersachsen, 2005 und 2007). (Quelle: Eigene Auswertung basierend auf Osterburg (2010))

Abb. 5 Anteil der Maisanbaufläche für Biogas an der Ackerfläche und Anteil der Grünlandumwandlung in Ackerland am Grünland 2005 in Abhängigkeit von der Entfernung zur nächsten Biogasanlage (Niedersachsen, 2005 und 2007). (Quelle: Eigene Auswertung basierend auf Osterburg (2010))
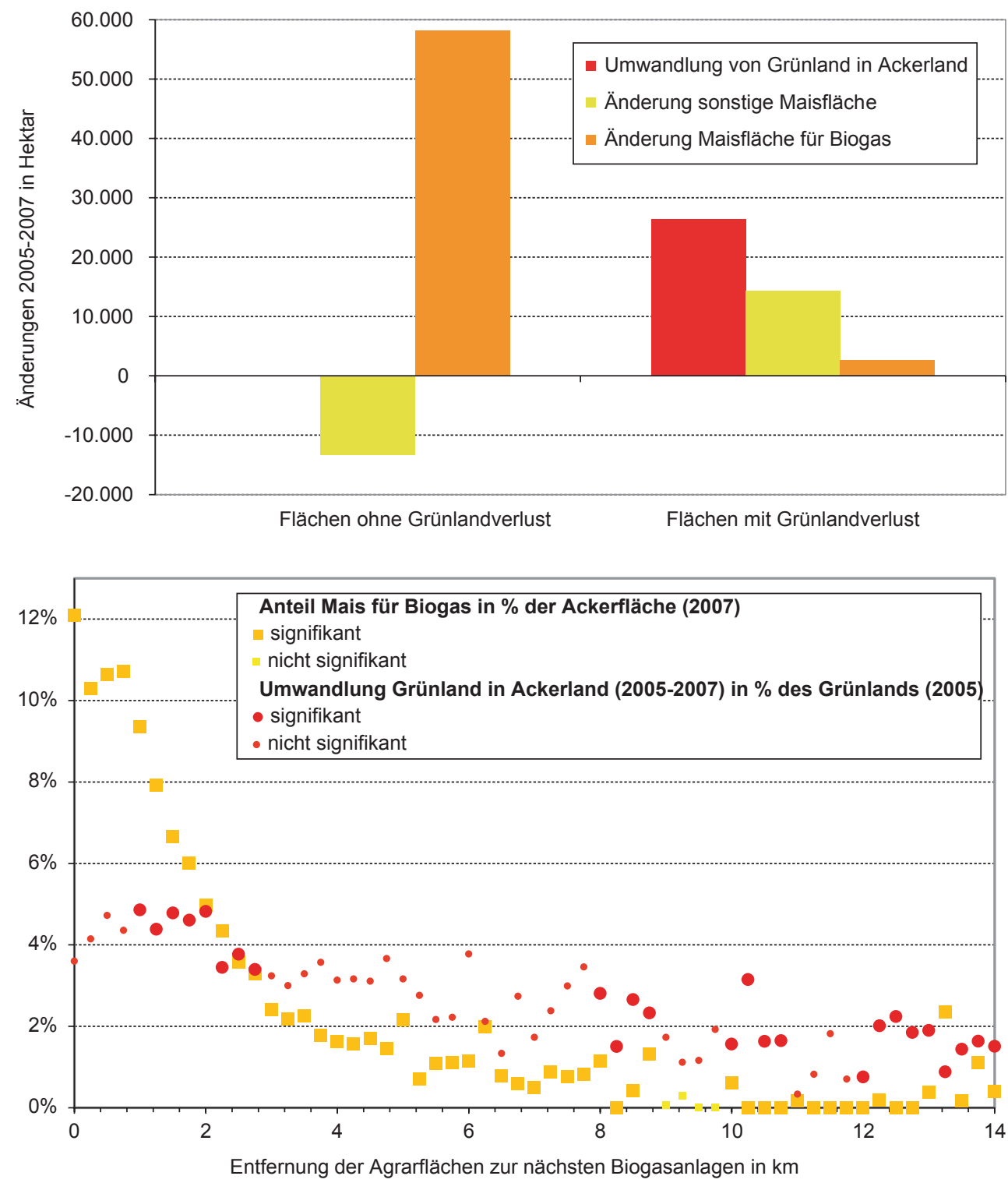

deutlich, dass die der Biogasproduktion dienende Maisfläche überwiegend auf der bereits bestehenden Ackerfläche ausgeweitet wurde. Die sonstige Maisfläche ist dagegen leicht zurückgegangen. Auf den Flächen mit Grünlandumwandlung wurde im Jahr 2007 auf über 60\% Mais angebaut, davon jedoch nur ein kleiner Teil als Gärsubstrat.

Die räumliche Nähe zur nächsten Biogasanlage dient als Indikator, um räumlich hoch aufgelöste Analysen zur Wirkung von Anlagen auf die Flächennutzung vorzunehmen, auch unabhängig davon, ob auf der jeweiligen Flächen Betriebe wirtschaften, die Pflanzen für Gärsubstrate anbauen (vgl. Abb. 5). Für den Anteil der Maisanbaufläche für Biogas ergibt sich im Logit-Modell erwartungsgemäß ein starker Zusammenhang mit der Nähe zu Biogasanlagen, der fast durchweg statistisch signifikant ist. Der zunächst stark positive Zusammenhang wird erst bei einer Entfernung von über $10 \mathrm{~km}$ negativ. Die Grünlandumwandlung weist dagegen einen vergleichsweise schwachen Zusammenhang mit der Nähe zu Biogasanlagen auf. Der Zusammenhang ist zwischen 1 und $3 \mathrm{~km}$ Entfernung signifikant positiv und bei über $8 \mathrm{~km}$ Entfernung meist signifikant negativ.

Aus der Analyse der räumlichen Lage von Flächen in Relation zu Biogasanlagen lassen sich demnach nur schwache Zusammenhänge ablesen. Dies lässt sich dadurch erklären, dass auch viele Betriebe ohne Gärsubstratanbau Grünland in Ackerland umwandeln. Um zu untersuchen, welche Betriebe an der Umwandlung von Grünland in Ackerland beteiligt sind, wurden die im Jahr 2007 im InVeKoS enthaltenen Betriebe nach Gärsubstratanbau und Art der Tierhaltung klassifiziert. Tabelle 3 zeigt, dass $75 \%$ der Grünlandumwandlung auf Betriebe ohne Gärsubstratproduktion entfallen, darunter allein knapp 58\% allein auf Betriebe mit Milchproduktion. Dies ist vor allem auf deren hohen Flächenanteil am gesamten Grünland zurück- 
Tab. 3 Grünlandumwandlung in Ackerland in Niedersachsen zwischen 2005 und 2007 in verschiedenen Betriebstypen mit und ohne Biogas-Gärsubstratanbau. (Quelle: Eigene Auswertung basierend auf Osterburg (2010))

\begin{tabular}{|c|c|c|c|c|c|c|}
\hline Betriebe & $\begin{array}{l}\text { Grünlandfläche } \\
\text { (2005) }\end{array}$ & $\begin{array}{l}\text { Anteil am ges. } \\
\text { Grünland (2005) } \\
(\%)\end{array}$ & $\begin{array}{l}\text { Anteil an ges. } \\
\text { Grünland- } \\
\text { umwandlung } \\
(2005-2007)(\%)\end{array}$ & $\begin{array}{l}\text { Grünlandum- } \\
\text { wandlung in \% }\end{array}$ & $*$ & $\begin{array}{l}\text { Davon nach Bewirt- } \\
\text { schafterwechsel (\%) }\end{array}$ \\
\hline \multicolumn{7}{|l|}{ Ohne Gärsubstratanbau } \\
\hline Ausscheidende Betriebe & 59.291 & 7,9 & - & - & & - \\
\hline Ohne Tierhaltung & 24.129 & 3,2 & 3,6 & 4,0 & a & 49,5 \\
\hline Schweine, Geflügel & 11.259 & 1,5 & 3,0 & 7,1 & $\mathrm{~b}$ & 56,6 \\
\hline Rinder ohne Milchkühe, Schafe, Pferde & 177.795 & 23,8 & 10,9 & 1,6 & $\mathrm{c}$ & 24,4 \\
\hline Milchproduktion extensiv** & 179.920 & 24,1 & 18,9 & 2,8 & d & 30,5 \\
\hline Milchproduktion intensiv** & 223.336 & 29,9 & 38,9 & 4,6 & $\mathrm{e}$ & 22,7 \\
\hline Insgesamt ohne Gärsubstratanbau & 616.439 & 90,5 & 75,2 & 3,0 & & 27,5 \\
\hline \multicolumn{7}{|l|}{ Mit Gärsubstratanbau } \\
\hline Ohne Tierhaltung & 3.354 & 0,4 & 2,3 & 18,7 & $\mathrm{f}$ & 57,6 \\
\hline Schweine, Geflügel & 3.017 & 0,4 & 2,2 & 19,1 & $\mathrm{f}$ & 63,7 \\
\hline Rinder ohne Milchkühe, Schafe, Pferde & 14.105 & 1,9 & 4,5 & 8,5 & g & 30,3 \\
\hline Milchproduktion extensiv** & 26.701 & 3,6 & 8,1 & 8,1 & g & 30,9 \\
\hline Milchproduktion intensiv** & 23.708 & 3,2 & 7,7 & 8,7 & $\mathrm{~h}$ & 31,0 \\
\hline Insgesamt mit Gärsubstratanbau & 70.885 & 9,5 & 24,8 & 9,3 & & 36,2 \\
\hline Insgesamt & 687.324 & 100 & 100 & 3,6 & & 29,7 \\
\hline
\end{tabular}

*Gleiche Buchstaben bedeuten, dass Unterschiede innerhalb der entsprechend gekennzeichneten Gruppen nicht signifikant sind $(P>0,05$, Wilcoxon Rangsummentest)

**Milchproduktion extensiv: $<1,8$ Großvieheinheiten $(\mathrm{GV})$ pro Hektar Hauptfutterfläche, Milchproduktion intensiv: $>=1,8$ GV pro Hektar Hauptfutterfläche

zuführen. Betriebe mit einer intensiven Milchproduktion weisen eine leicht überdurchschnittliche Rate bei der Grünlandumwandlung auf. Nur etwa $25 \%$ des in Ackerland umgewandelten Grünlands finden sich in Betrieben mit Gärsubstratanbau. Bei diesen Betrieben ist die Rate der Grünlandumwandlung im Schnitt mehr als dreimal höher als bei Betrieben ohne Gärsubstratanbau.

$\mathrm{Ob}$ die in Tab. 3 betrachteten Betriebe die Grünlandflächen selbst umgewandelt haben, lässt sich in vielen Fällen nicht feststellen, da etwa $30 \%$ aller Grünlandumwandlungen in Ackerland im Zusammenhang mit einem Bewirtschafterwechsel stehen. Bei Übergabe von Grünlandflächen an andere Betriebe besteht demnach ein besonders hohes Risiko für Grünlandverluste.

\section{Diskussion}

Die Ergebnisse zeigen, dass räumlich hoch aufgelöste Daten erheblich helfen, die Prozesse, die zu Landnutzungsänderungen führen, besser zu verstehen. Ein Kennzeichen der landwirtschaftlichen Landnutzung ist, dass gegenläufige Prozesse gleichzeitig auf benachbarten Flächen ablaufen können. Ein Beispiel hierfür ist die Umwandlung von Grünland zu Ackerland und umgekehrt. Dies führt dazu, dass mit zunehmender räumlicher Auflösung der feststellbare Bruttoumfang des in Ackerland umgewandelten Grünlandes zunimmt, da die Auswirkungen der Saldierung von
Umwandlungsgewinnen mit Verlusten innerhalb derselben räumlichen Einheit zurückgehen. Eine ausschließliche Betrachtung der Netto-Veränderung der Grünlandfläche unterschätzt die Umweltwirkungen des erfolgten Landnutzungswandels, da langjähriges Dauergrünland sowohl aus Gründen des Arten- und Biotopschutzes (Dierschke/Briemle 2002) als auch bezüglich des Erhalts des Bodenkohlenstoffs (Poeplau/Don/Vesterdal et al. 2011) meist wertvoller ist als neu etabliertes Grünland. Nach Rothe/Osterburg/de Witte et al. (2010: 191) hat über die Hälfte des Grünlands in Deutschland aufgrund von Schutzstatus, Bodenverhältnissen oder Hanglage einen hohen naturschutzfachlichen Stellenwert.

Grünland wird insbesondere dann in Ackerland umgewandelt, wenn es in einen neuen Betrieb übergeht. Ursache hierfür ist, dass der aufnehmende Betrieb keine Verwendungsmöglichkeit für die Aufwüchse hat (viehlose Betriebe) oder eine alternative Ackernutzung für ihn wirtschaftlicher ist (Milchvieh- oder Biogasbetriebe). Aufgrund des mit der Flächenübergabe verbundenen, hohen Umwandlungsrisikos ist die Wirksamkeit betrieblicher Ansätze zum Grünlandschutz sehr begrenzt. Bei betrieblichen Regelungen (z. B. Cross Compliance für das EU-Förderrecht) ist nach Flächenübergaben nicht eindeutig nachvollziehbar, ob der Flächeneigentümer oder der abgebende bzw. aufnehmende Betrieb für die Grünlandumwandlung verantwortlich ist. Ferner kann bei der Feststellung eines Verstoßes oft nur der aufnehmende Betrieb belangt werden, nicht jedoch die anderen Beteiligten (Vorbesitzer und Flächeneigentümer). 
Auch produktspezifische Zertifizierungsregeln sind nicht zielführend. So schließt die Biomassestrom-Nachhaltigkeitsverordnung (BioStNachV) die Förderung von nachwachsenden Rohstoffen für flüssige Energieträger aus, die auf umgewandelten Grünlandflächen erzeugt werden. Selbst wenn diese Regelung auf Rohstoffe für gasförmige Energieträger erweitert würde, wäre damit für den Erhalt des Grünlandes wenig gewonnen. So ist in Niedersachsen zwar Mais die Hauptfrucht nach der Umwandlung von Grünland. Der Anbau von Gärsubstraten erfolgt aber zum weit überwiegenden Teil auf schon bestehender Ackerfläche und nur zu einem kleinen Teil auf umgewandelter Grünlandfläche. Der Großteil des auf umgewandeltem Grünland angebauten Silomaises wird durch Milchviehbetriebe ohne Biogasanlagen genutzt. In Milchviehbetrieben ermöglichen höhere Maisanteile, die Milchleistung pro Kuh zu steigern und mehr Tiere zu halten. Die Geest entlang der Nordsee ist die Region mit den flächig höchsten Grünlandverlusten und der stärksten Ausdehnung des Maisanbaus und gleichzeitig auch diejenige mit dem stärksten Wachstum der Milcherzeugung (Schramek/Osterburg/Kasperczyk et al. 2012). Insbesondere Betriebe, die Mais sowohl als Futtermittel als auch als Gärsubstrat verwenden, können Zertifizierungsanforderungen durch eine geänderte Zuteilung der Silomaisflächen auf die Tierhaltung bzw. Biogaserzeugung umgehen. Im Extremfall führen solche Regelungen nur zu einem erhöhten Transportaufwand, da identische Produkte zwischen unterschiedlichen Betrieben oder Regionen hinund hertransportiert werden. ${ }^{4}$

In Bezug auf die Grünlandumwandlung scheinen im betrachteten Zeitraum indirekte Effekte (z. B. aufgrund steigender Pachtpreise) von geringerer Bedeutung zu sein, da die Umwandlungsrate nur schwach mit der Nähe zu Biogasanlagen korreliert. Allerdings ist bei der Maisfläche, die nicht zur Erzeugung von Gärsubstraten genutzt wird, eine Verlagerung von der bestehenden Ackerfläche auf umgewandelte Grünlandflächen festzustellen. Aufgrund der dargestellten Bedeutung von Verdrängungs- und Verlagerungseffekten ist die von Starick/Klöckner/Möller et al. (2011) geforderte Abgrenzung von Ausschlussräumen für den Bioenergieanbau genauso wie die von Greiff/Weber-Blaschke/Faulstich et al. (2010) vorgeschlagene regionale Differenzierung der Förderhöhe wenig zielführend. Ein wirksamer Schutz bestimmter Lebensräume ist nur flächenspezifisch möglich und muss für alle Fälle einer Landnutzungsänderung gelten. Wie dargelegt, ermöglichen die neuen Datenquellen

\footnotetext{
${ }^{4}$ So führen unterschiedliche Standards für die nachzuweisende Treibhausgasminderung von Bioethanol dazu, dass Brasilien große Mengen Ethanol aus Zuckerrohr in die USA exportiert und gleichzeitig aus den USA erhebliche Mengen Ethanol aus Körnermais importiert (Meyer/ Schmidhuber/Barreiro-Hurlé 2012). Hierbei ist anzumerken, dass das Ethanol in beiden Fällen über die gleichen physikalischen und chemischen Produkteigenschaften verfügt.
}

eine sehr differenzierte Analyse von Landnutzungsänderungen im Agrarbereich. Allerdings sind mit der Verarbeitung dieser Daten auch neue Herausforderungen verknüpft. Hier sind organisatorische Aspekte von inhaltlichen Punkten zu trennen.

Die Beschaffung und Verwaltung der Geofachdaten ist mit einem hohen zeitlichen Aufwand verbunden. Bundesweit vorliegende Daten sind zwar meist relativ gut dokumentiert und zugänglich, zum Teil auch ohne konkreten Projektbezug oder Zeitbeschränkung. Die meisten Fachdaten werden allerdings von den Ländern bereitgestellt, und hier gibt es zum Teil größere Hürde zu überwinden. So fehlt in den Bundesländern oft eine zentrale Stelle, die alle verfügbaren Geofachdaten verwaltet und sie Dritten zur Verfügung stellt. Infolgedessen sind aufwendige Recherchen bei den einzelnen Fachbehörden notwendig. Ferner sind die Nutzungsbedingungen zum Teil einzelfallbezogen auszuhandeln, denn sie beinhalten oft eine projektgebundene, sehr enge zeitliche und inhaltliche Beschränkung der Nutzungsrechte. Auch unterscheiden sich die Anforderungen für eine Verlängerung des Nutzungszeitraumes oder die Erweiterung der Nutzungsrechte deutlich. Positiv zu erwähnen sind die Initiativen einzelner Bundesländer (LfU 2013a; LfU 2013b; LUBW 2013; MUGV 2013), die Teile ihrer Geofachdaten übersichtlich darstellen und zum direkten Download zur Verfügung stellen. Es ist zu hoffen, dass durch die INSPIRE-Richtlinie (Infrastructure for Spatial Information in Europe) zukünftig Beschaffung, Verwaltung und Aufbereitung von Geofachdaten weniger zeitintensiv wird. INSPIRE soll die grenzübergreifende Nutzung von Geofachdaten erleichtern und eine einheitliche Beschreibung der Daten sowie einen einfachen Onlinezugang zu den Daten gewährleisten (INSPIRE 2007).

Neben dem Datenzugang ist auch deren Aufbereitung sehr zeitaufwendig. Es existieren zwar länderübergreifende Arbeitsgruppen, aber einheitliche Standards sind noch wenig verbreitet, was die inhaltliche Ausrichtung der Geofachdaten (Auswahl und Strukturierung der aufgenommenen Inhalte, Ausprägung der Attribute) sowie ihre räumliche und zeitliche Auflösung betrifft. Diese Unterschiede in der Datenbasis erschweren den Vergleich zwischen einzelnen Bundesländern, und eine bundesweite Analyse ist nur eingeschränkt möglich. Auch bei den InVeKoS-Daten findet sich das Problem der Datenheterogenität. Im Rahmen der Vorgaben der InVeKoSV (2004) wurde die Abgrenzung der FLIK-Flächen in den einzelnen Bundesländern unterschiedlich umgesetzt (Feldblock, Schlag, Feldstück, Flurstück). Bei den auf Feldblöcken und Flurstücken basierenden Flächenidentifizierungssystemen können definitionsbedingt verschiedene Nutzungen auf eine FLIK-Fläche gebucht werden. Daraus können sich besonders im Zeitreihenvergleich Unsicherheiten bei der Identifizierung und Quantifizierung von Landnutzungsänderungen ergeben. 
Eine inhaltliche Herausforderung ist die Verarbeitung von Informationen, die räumlich oder inhaltlich unterschiedlich stark aggregiert sind. Hier sind im Konkreten drei Problembereiche zu differenzieren. Erstens ist die unterschiedliche Wahrscheinlichkeit der fehlerhaften Zuweisung von Objekteigenschaften zu beachten (z. B. Bodentyp) (Lösel 2005). Je größer der Erfassungsmaßstab ist, desto geringer sollte dieser Fehler sein. Zweitens kann allein die unterschiedliche Aggregation von Fachdaten zu abweichenden Ergebnissen bei der statistischen Analyse führen. Dieses Problem ist hinlänglich unter dem Begriff „modifiable area unit problem“ (MAUP) bekannt (Openshaw/Taylor 1979). Drittens entstehen durch Lagefehler der Polygongrenzen Fehler- oder Splitterpolygone (spurious or sliver polygons) an den Polygonrändern (Zhang/Goodchild 2003). Diese Polygone fallen insbesondere bei der Analyse von Landnutzungsänderungen ins Gewicht, da hier Zeitreihen benötigt werden. So kann die Ursache der Verschiebung der Polygongrenze über die Zeit entweder eine tatsächliche Nutzungsänderung oder eine unterschiedliche Digitalisierung der Polygongrenze sein. In letzterem Fall werden Landnutzungsänderungen quantifiziert, die nicht stattgefunden haben. Gegenwärtig wird an Algorithmen gearbeitet, um bei widersprüchlicher Information der Datengrundlage die Qualität der Ausgangsdaten stärker zu berücksichtigen.

Vor dem Hintergrund der Vergleichbarkeit der Daten und der Möglichkeit zur bundesweiten Analyse wären eine stärkere Standardisierung der Datensätze (Aufnahmejahr, erhobene Attributdaten und ihre Ausprägung) und eine gewisse zeitliche Kontinuität der erhobenen Merkmale wünschenswert. Bisher ist dies nur für einige bundesweit vorliegende Daten wie das Basis-DLM gegeben. Ferner sollten Möglichkeiten geschaffen werden, die InVeKoS-Daten, die den im Augenblick besten Datensatz zur landwirtschaftlichen Flächennutzung darstellen, vermehrt für wissenschaftliche Analysen nutzen zu können.

Die in diesem Beitrag vorgestellten Analysen sind nur ein Beispiel für die unterschiedlichen Auswertungsmöglichkeiten, die auf der Grundlage der neuen, georeferenzierten Datenbasis zur landwirtschaftlichen Landnutzung möglich sind. Weitere Auswertungen betreffen die Evaluierung von flächenbezogenen Agrarumweltmaßnahmen bezüglich ihrer räumlichen Verteilung und Konzentration in Schutz- oder Zielgebieten, die Analyse von Fruchtfolgen und die Analyse des betrieblichen Strukturwandels und seiner Auswirkungen auf die Flächennutzung.

\section{Literatur}

Anselin, L.; Lozano, N.; Koschinsky, J. (2006): Rate Transformation and Smoothing. Urbana, Illinois. http://geodacenter.asu.edu/pdf/ smoothing_06.pdf (12.12.2013).
Bakker, J. P.; Berendse, F. (1999): Constraints in the restoration of ecological diversity in grassland and heathland communities. In: Trends in Ecological Evolution 14 (2), 63-68.

DBFZ - Deutsches BiomasseForschungsZentrum (2011): Nachhaltige Biogaserzeugung in Deutschland - Bewertung der Wirkungen des EEG. Leipzig.

de Witte, T. (2012): Entwicklung eines betriebswirtschaftlichen Ansatzes zur Ex-ante-Analyse von Agrarstrukturwirkungen der Biogasförderung - angewendet am Beispiel des EEG 2009 in Niedersachsen. Braunschweig. = Landbauforschung Sonderheft 366.

Dierschke, H.; Briemle, D. (2002): Kulturgrasland. Wiesen, Weiden und verwandte Staudenfluren. Stuttgart.

Drösler, M.; Adelmann, W.; Augustin, J.; Bergman, L.; Beyer, C.; Chojnicki, B.; Förster, C.; Freibauer, A.; Giebels, M.; Görlitz, S.; Höper, H.; Kantelhardt, J.; Liebersbach, H.; Hahn-Schöfl, M.; Minke, M.; Petschow, U.; Pfadenhauer, J.; Schaller, L.; Schägner, P.; Sommer, M.; Thuille, A.; Wehrhan, M. (2013): Klimaschutz durch Moorschutz. Schlussbericht des Vorhabens „KlimaschutzMoornutzungsstrategien“ 2006-2010. Freising. http://edok01.tib. uni-hannover.de/edoks/e01fb13/735500762.pdf (10.12.2013).

DVL - Deutscher Verband für Landschaftspflege; NABU (Naturschutzbund) (2007): Bioenergie? - Aber natürlich! Nachwachsende Rohstoffe aus Sicht des Umwelt- und Naturschutzes. Ansbach/ Berlin. $=$ Landschaft als Lebensraum 12.

EEA - European Environment Agency (2007): Estimating the environmentally compatible bioenergy potential from agriculture. Kopenhagen. $=$ EEA Technical Report 12/2007.

Emmann, C. H.; Theuvsen, L. (2012): Einfluss der Biogasproduktion auf den regionalen Pachtmarkt. In: Berichte über Landwirtschaft 90 (1), 84-113.

Europäische Kommission (2009): Verordnung (EG) Nr. 1122/2009 der Kommission mit Durchführungsbestimmungen zur Verordnung (EG) Nr. 73/2009 des Rates hinsichtlich der Einhaltung anderweitiger Verpflichtungen, der Modulation und des Integrierten Verwaltungs- und Kontrollsystems im Rahmen der Stützungsregelungen für Inhaber landwirtschaftlicher Betriebe gemäß der genannten Verordnung und mit Durchführungsbestimmungen zur Verordnung (EG) Nr. 1234/2007 hinsichtlich der Einhaltung anderweitiger Verpflichtungen im Rahmen der Stützungsregelungen für den Weinsektor. Brüssel.

Europäischer Rat (2009): Verordnung (EG) Nr. 73/2009 des Rates vom 19. Januar 2009 mit gemeinsamen Regeln für Direktzahlungen im Rahmen der gemeinsamen Agrarpolitik und mit bestimmten Stützungsregelungen für Inhaber landwirtschaftlicher Betriebe und zur Änderung der Verordnungen (EG) Nr. 1290/2005, (EG) Nr. 247/2006, (EG) Nr. 378/2007 sowie zur Aufhebung der Verordnung (EG) Nr. 1782/2003. Brüssel.

Freibauer, A.; Drösler, M.; Gensior, A.; Schulze, E.-D. (2009): Das Potenzial von Wäldern und Mooren für den Klimaschutz in Deutschland und auf globaler Ebene. In: Natur und Landschaft 84 (1), 20-25.

Gawel, E.; Ludwig, G. (2011): Indirekte Landnutzungsänderungen durch Energiepflanzenanbau - Erfassungsmethoden und ihre Bewertung. In: Naturschutz und Landschaftsplanung 43 (8), 237-243.

Gocht, A.; Röder, N. (2011): Salvage the treasure of geographic information in farm census data. Vortrag im Rahmen des EAAE 2011 Congress "Change and Uncertainty, Challenges for Agriculture, Food and Natural Resources", 30.08.2011-02.09.2011. Zürich. http://ideas.repec.org/p/ags/eaae11/114824.html (11.12.2013).

Greiff, K. B.; Weber-Blaschke, G.; Faulstich, M.; von Haaren, C. (2010): Förderung eines umweltschonenden Energiepflanzenanbaus. Vorschlag für eine raum- und energiepflanzendifferenzierte Prämie. In: Naturschutz und Landschaftsplanung 42 (4), 101-106.

Habermann, H.; Breustedt, G. (2011): Einfluss der Biogaserzeugung auf landwirtschaftliche Pachtpreise in Deutschland. In: German Journal of Agricultural Economics 60 (2), 85-100. 
Hübner, R. (2010): Auswirkungen nachwachsender Rohstoffe zur Energieerzeugung auf Natur und Landschaft in Bayern - unter Berücksichtigung wasserwirtschaftlicher Aspekte. Freising. http://wdl. wzw.tum.de/forschung/publikationen/download/huebner2010 endbericht_nawaro_2010.pdf (10.12.2013).

INSPIRE (2007): Richtlinie 2007/2/EG des Europäischen Parlaments und des Rates vom 14. März 2007 zur Schaffung einer Geodateninfrastruktur in der Europäischen Gemeinschaft (INSPIRE). Brüssel.

InVeKoSV (2004): Verordnung über die Durchführung von Stützungsregelungen und des Integrierten Verwaltungs- und Kontrollsystems vom 3. Dezember 2004, die zuletzt durch Artikel 2 der Verordnung vom 15. Dezember 2011 geändert worden ist.

IPCC - Intergovernmental Panel on Climate Change (2000): Land Use, Land-Use Change, and Forestry. Cambridge.

Johnston, A. E.; Poulton, P. R.; Coleman, K. (2009): Soil organic matter: Its importance in sustainable agricultural and carbon dioxide fluxes. In: Sparks, D. L. (Hrsg.): Advances in Agronomy. New York, 1-57.

JRC - Joint Research Centre (2012): Orthoimage technical specifications for the purpose of LPIS. http://marswiki.jrc.ec.europa. eu/wikicap/index.php/Orthoimage_technical_specifications_for the purpose of LPIS (10.12.2013).

Kosfeld, R.; Gückelhorn, F. (2012): Ökonomische Effekte erneuerbarer Energien auf regionaler Ebene. In: Raumforschung und Raumordnung 70 (5), 437-449.

Kretschmer, B.; Bowyer, C.; Buckwell, A. (2012): EU Biofuel Use and Agricultural Commodity Prices: A Review of the Evidence Base. London.

Laggner, B.; Orthen, N. (2013): A new GIS toolbox for integrating massive heterogeneous GIS data for land use analysis. http:// elogeo.nottingham.ac.uk/xmlui/bitstream/handle/url/195/ FOSS4G13_LaggnerOrthen_GIS_LandUseAnalysis.pdf?sequen$\mathrm{ce}=1(12.12 .2013)$.

Lal, R. (2008): Carbon sequestration. In: Philosophical Transactions of the Royal Society B 363 (1492), 815-830.

LfU - Bayerisches Landesamt für Umwelt (2013a): Biotopflächen und Sachdaten. http://www.lfu.bayern.de/natur/biotopkartierung daten/daten_download/index.htm (12.12.2013).

LfU - Bayerisches Landesamt für Umwelt (2013b): Natura 2000 in Bayern. Abgrenzungen. http://www.lfu.bayern.de/natur/natura2000_ abgrenzungen/index.htm (12.12.2013).

Lösel, G. (2005): Informationsgüte kleinmaßstäbiger Bodenkarten. Probleme und Entwicklungsmöglichkeiten. Dissertation an der Universität Hannover.

LUBW - Landesanstalt für Umwelt, Messungen und Naturschutz Baden-Württemberg (2013): Daten- und Kartendienst, Geodatenserver. http://www.lubw.baden-wuerttemberg.de/servlet/is/41531/ (31.01.2014).

Meyer, S.; Schmidhuber, J.; Barreiro-Hurlé, J. (2012): Intra-industry trade in biofuels: how environmental legislation fuels resource use and $\mathrm{GHG}$ emissions. Rom. $=$ ESA Working Paper 12-08.

ML - Niedersächsisches Ministerium für Ernährung, Landwirtschaft, Verbraucherschutz und Landesentwicklung; MU - Niedersächsisches Ministerium für Umwelt, Energie und Klimaschutz (2012): Biogas in Niedersachsen. Entwicklung, Stand und Perspektiven. Hannover.

MUGV - Ministerium für Umwelt, Gesundheit und Verbraucherschutz des Landes Brandenburg (2013): Geoinformationen. http:// www.mugv.brandenburg.de/cms/detail.php/bb1.c.280662.de (12.12.2013).

Nitsch, H.; Osterburg, B.; von Buttlar, C.; von Buttlar, H.-B. (2008): Aspekte des Gewässerschutzes und der Gewässernutzung beim Anbau von Energiepflanzen. Ergebnisse eines Forschungsvorhabens im Auftrag des Umweltbundesamtes. Braunschweig. = Arbeitsberichte aus der vTI-Agrarökonomie 03/2008.
Openshaw, S.; Taylor, P.J. (1979): A million or so correlation coefficients: Three experiments on the modifiable areal unit problem. In: Wrigley, N. (Hrsg.): Statistical applications in the spatial sciences. London, 127-144.

Osterburg, B. (2010): Wirkungen von Biogasanlagen auf die Flächennutzung in Niedersachsen - eine Analyse von Daten des Integrierten Verwaltungs- und Kontrollsystems (InVeKoS ). Projektbericht. Braunschweig.

Osterburg, B.; Nitsch, H.; Laggner, B.; Roggendorf, W. (2009): Auswertung von Daten des Integrierten Verwaltungs- und Kontrollsystems zur Abschätzung von Wirkungen der EU-Agrarreform auf Umwelt und Landschaft. Braunschweig. = Arbeitsberichte aus der vTI-Agrarökonomie 07/2009.

Poeplau, C.; Don, A.; Vesterdal, L.; Leifeld, J.; van Wesemael, B.; Schumacher, J.; Genisor, A. (2011): Temporal dynamics of soil organic carbon after land-use change in the temperate zone - carbon response functions as a model approach. In: Global Change Biology 17 (7), 2415-2427.

Pölking, A.; Stiepel, B.; Premke-Kraus, M.; Will, J.; Lüdtke, S.; Oppermann, R.; Baumann, A. (2006): Bioenergie und Biogasförderung nach dem neuen EEG und ihre Auswirkungen auf Natur und Landschaft. Wolfenbüttel.

Röder, N.; Gocht A. (2013): Recovering localised information on agricultural structures while observing data confidentiality regulations - the potential of different data aggregation and segregation techniques. In: Journal of Land Use Science 8 (1), 31-46.

Roskam, A. (2007): Bioenergie und Grundwasserschutz. Nutzungskonflikte und Zusammenarbeit in Ostfriesland - Praktische Beispiele. Energieeffizienz im Vergleich. Vortrag bei der Fachtagung der Alfred Toepfer Akademie für Naturschutz am 31. Mai 2007 im Camp Reinsehlen.

Rothe, A.; Osterburg, B.; de Witte, T.; Zimmer, Y. (2010): Endbericht: Modellgestützte Folgenabschätzungen für den Anbau nachwachsender Rohstoffe in Deutschland. Braunschweig. http://iteratur. vti.bund.de/digbib_extern/dn047291.pdf (12.12.2013).

Schramek, J.; Osterburg, B.; Kasperczyk, N.; Nitsch, H.; Wolff, A.; Weis, M.; Hülemeyer, K. (2012): Vorschläge zur Ausgestaltung von Instrumenten für einen effektiven Schutz von Dauergrünland. Bonn. http://www.bfn.de/fileadmin/MDB/documents/service/ Skript_323.pdf (10.12.2013).

Searchinger, T.; Heimlich, R.; Houghton, R.; Dong, F.; Elobeid, A.; Fabiosa, J.; Tokgoz, S.; Hayes, D.; Yu, T.-H. (2008): Use of U.S. Croplands for Biofuels Increases Greenhouse Gases through Emissions from Land-Use Change. In: Science 319 (5867), 1238-1240.

Starick, A.; Klöckner, K.; Möller, I.; Gaasch, N.; Müller, K. (2001): Entscheidungshilfen für eine nachhaltige räumliche Entwicklung der Bioenergiebereitstellung - Methoden und ihre instrumentelle Anwendung. In: Raumforschung und Raumordnung 69 (6), 367-382.

Statistisches Bundesamt (2013): Flächenerhebung nach Art der tatsächlichen Nutzung 1992-2013. Wiesbaden.

Stoate, C.; Baldi, A.; Beja, P.; Boatman, N. D.; Herzon, I.; van Doorn, A.; de Snoo, G. R.; Rakosy, L.; Ramwell, C. (2009): Ecological impacts of early 21 st century agricultural change in Europe - A review. In: Journal of Environmental Management 91 (1), 22-46.

WBGU - Wissenschaftlicher Beirat der Bundesregierung Globale Umweltveränderungen (2009): Welt im Wandel - Zukunftsfähige Biomasse und nachhaltige Landnutzung. Berlin.

Zhang, J.; Goodchild, M. F. (2003): Uncertainty in geographical information. London. 